NUREG-1214

Revision 14

\title{
Historical Data Summary of the Systematic Assessment of Licensee Performance
}

Manuscript Completed: October 1996

Date Published: October 1996

D. Gamberoni

Division of Inspection and Support Programs

Office of Nuclear Reactor Regulation

U.S. Nuclear Regulatory Commission

Washington, DC 20555-0001

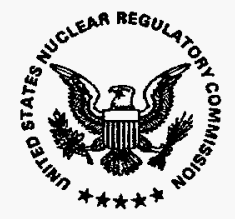




\section{DISCLAMMER}

Portions of this document may be illegible in electronic image products. Images are produced from the best available original document. 


\section{DISCLAIMER}

This report was prepared as an account of work sponsored by an agency of the United States Government. Neither the United States Government nor any agency thereof, nor any of their employees, makes any warranty, express or implied, or assumes any legal liability or responsibility for the accuracy, completeness, or usefulness of any information, apparatus, product, or process disclosed, or represents that its use would not infringe privately owned rights. Reference herein to any specific commercial product, process, or service by trade name, trademark, manufacturer, or otherwise does not necessarily constitute or imply its endorsement, recommendation, or favoring by the United States Government or any agency thereof. The views and opinions of authors expressed herein do not necessarily state or reflect those of the United States Government or any agency thereof. 


\section{ABSTRACT}

The Historical Data Summary of the Systematic Assessment of Licensee Performance (SALP) is produced periodically by the U.S. Nuclear Regulatory Commission. This summary provides the results of the SALP assessments for each facility by NRC region and is further divided into two sections. Section 1 presents the most recent SALP report ratings for facilities in operation. Section 2 presents a chronological listing of all SALP report ratings for each operating facility with a final report of August 1988 or 1ater. 



\section{CONTENTS}

\section{Page}

Introduction

Section 1, SALP Summary: Most Recent Ratings

1-1

Section 2, SALP History: Facilities in Operation

2-1 
INTRODUCTION

\section{Organization}

The Historical Data Summary of the Systematic Assessment of Licensee Performance (SALP) is produced periodically by the U.S. Nuclear Regulatory Commission (NRC). This revision of the summary provides the results of SALP. assessments for reports issued during the period January 1, 1994 through September 30,1996 . The results are presented for each facility in two sections and are further divided by NRC region. Section 1 presents the most recent SALP report ratings for facilities in operation. Section 2 presents a chronological listing of all SALP report ratings for each operating facility since August 1988. Earlier revisions of this NUREG contain the SALP ratings prior to August 1988 and for plants under construction.

\section{Background}

The SALP program is an integrated NRC staff effort to collect and evaluate observations and data to assess and better understand the reasons for a 1 icensee's performance. The program is described in NRC Management Directive 8.6, "Systematic Assessment of Licensee Performance," which was issued in July 1993. The purpose of the program is to assess licensee performance in selected functional areas. For facilities in operation that have been evaluated since the issuance of Management Directive 8.6 in July 1993, the functional areas include operations (OPS), maintenance (MAINT), engineering (ENG), and plant support (PS). The plant support functional area includes radiological controls, emergency preparedness, and security issues. The program was previously described in NRC Manual Chapter 0516. From June 1988 until July 1993, the functional areas included plant operations (OPS), maintenance/surveillance (MAINT), engineering/technical support (ENG), radiological controls (RADCON), emergency preparedness (EP), security (SEC), and safety assessment/quality verification. In this version of NUREG-1214, the previous separate SALP scores for radiological controls, emergency preparedness, and security are displayed together under the plant support heading in the following order: RADCON/EP/SEC. Prior to June 1988 there were eleven functional areas.

Based on a review of the consolidated information, each functional area is placed into one of three performance categories. A Category 1 rating designates a superior level of safety performance. A Category 2 rating designates a good level of performance. A Category 3 rating designates an acceptable level of performance where NRC will consider increased levels of inspection effort. An " $N$ " indicates that no rating was given for that category because of insufficient information.

It is the policy of the U.S. Nuclear Regulatory Commission to use the SALP process to articulate the agency's observations and insights on the licensee's safety performance. The SALP report communicates those observations and insights to licensee management and the public. The NRC discourages use of SALP data for any purpose other than its intended objectives. Statistical or numerical balancing of data is inappropriate. 
Section 1

SALP Summary: Most Recent Ratings

NUREG-1214 


\section{SALP SUMMARY \\ REGION 1}

\section{(REACTORS IN OPERATION)}

\begin{tabular}{|c|c|c|c|c|c|}
\hline PLANT NAME & RPT & OPS & MAINT & ENG & PS \\
\hline BEAVER VALLEY $1 / 2$ & $07 / 95$ & 2 & 1 & 2 & 1 \\
\hline CALVERT CLIFFS $1 / 2$ & $07 / 95$ & 1 & 2 & 1 & 2 \\
\hline FITZPATRICK & $12 / 95$ & 2 & 2 & 2 & 2 \\
\hline GINNA & $05 / 95$ & 1 & 2 & 1 & 2 \\
\hline HADDAM NECK & $11 / 94$ & 2 & 2 & 2 & 1 \\
\hline HOPE CREEK 1 & $06 / 95$ & 2 & 2 & 2 & 1 \\
\hline INDIAN POINT 2 & $10 / 95$ & 2 & 1 & 1 & 2 \\
\hline INDIAN POINT 3 & $04 / 96$ & 3 & 2 & 3 & 1 \\
\hline LIMERICK $1 / 2$ & $05 / 95$ & 1 & 1 & 1 & 1 \\
\hline MAINE YANKEE & $10 / 95$ & 1 & 2 & 1 & 2 \\
\hline MILLSTONE $1 / 3$ & $08 / 94$ & 2 & 2 & 2 & 2 \\
\hline MILLSTONE 2 & $08 / 94$ & 3 & 3 & 2 & 2 \\
\hline NINE MILE POINT $1 / 2$ & $07 / 96$ & 1 & 2 & 2 & 2 \\
\hline OYSTER CREEK & $08 / 95$ & 1 & 2 & 1 & 2 \\
\hline PEACH BOTTOM $2 / 3$ & $12 / 95$ & 1 & 1 & 2 & 1 \\
\hline PILGRIM 1 & $05 / 96$ & 2 & 2 & 1 & 2 \\
\hline SALEM $1 / 2$ & $01 / 95$ & 3 & 3 & 2 & 1 \\
\hline SEABROOK 1 & $06 / 96$ & 1 & 2 & 1 & 1 \\
\hline SUSQUEHANNA $1 / 2$ & $09 / 95$ & 1 & 1 & 1 & 1 \\
\hline THREE MILE ISLAND 1 & $09 / 96$ & 1 & 1 & 2 & 2 \\
\hline VERMONT YANKEE & $08 / 95$ & 2 & 2 & 2 & 1 \\
\hline
\end{tabular}




\section{SALP SUMMARY}

REGION 2

\section{(REACTORS IN OPERATION)}

\begin{tabular}{llcccc} 
PLANT NAME & RPT & OPS & MAINT & ENG & PS \\
\hline BROWNS FERRY 2 & $04 / 95$ & 2 & 2 & 2 & 1 \\
BRUNSWICK 1/2 & $06 / 95$ & 1 & 1 & 1 & 1 \\
CATAWBA 1/2 & $11 / 95$ & 2 & 2 & 2 & 2 \\
CRYSTAL RIVER 3 & $10 / 95$ & 2 & 2 & 2 & 1 \\
FARLEY 1/2 & $05 / 95$ & 1 & 2 & 1 & 1 \\
HARRIS 1 & $04 / 96$ & 1 & 1 & 1 & 1 \\
HATCH 1/2 & $07 / 95$ & 2 & 2 & 1 & 2 \\
MCGUIRE 1/2 & $09 / 95$ & 2 & 2 & 1 & 1 \\
NORTH ANNA 1/2 & $01 / 95$ & 1 & 1 & 1 & 1 \\
OCONEE 1/2/3 & $06 / 96$ & 1 & 2 & 1 & 1 \\
ROBINSON 2 & $07 / 95$ & 2 & 2 & 2 & 2 \\
SEQUOYAH 1/2 & $09 / 96$ & 2 & 2 & 2 & 2 \\
ST LUCIE 1/2 & $02 / 96$ & 2 & 2 & 1 & 1 \\
SUMMER & $03 / 95$ & 1 & 1 & 2 & 1 \\
SURRY 1/2 & $02 / 95$ & 1 & 2 & 1 & 1 \\
TURKEY POINT 3/4 & $09 / 94$ & 1 & 1 & 1 & 1 \\
VOGTLE 1/2 & $03 / 96$ & 1 & 1 & 1 & 2 \\
WATTS BAR 1 & $\times X / X X$ & $X$ & $X$ & $X$ \\
\end{tabular}


SALP SUMMARY

REGION 3

(REACTORS IN OPERATION)

\begin{tabular}{|c|c|c|c|c|c|}
\hline PLANT NAME & RPT & OPS & MAINT & ENG & PS \\
\hline BIG ROCK POINT & $01 / 96$ & 2 & 2 & 2 & 1 \\
\hline BRAIDWOOD $1 / 2$ & $11 / 95$ & 2 & 2 & 2 & 1 \\
\hline BYRON $1 / 2$ & $09 / 94$ & 1 & 1 & 1 & 1 \\
\hline CLINTON & $08 / 95$ & 2 & 1 & 2 & 1 \\
\hline $\operatorname{coOK} 1 / 2$ & $07 / 96$ & 2 & 2 & 2 & 1 \\
\hline DAVIS-BESSE & $03 / 95$ & 1 & 1 & 1 & 1 \\
\hline DRESDEN $2 / 3$ & $04 / 95$ & 3 & 2 & 3 & 2 \\
\hline DUANE ARNOLD & $12 / 95$ & 1 & 2 & 1 & 1 \\
\hline FERMI 2 & $05 / 96$ & 3 & 2 & 2 & 1 \\
\hline KEWAUNEE & $03 / 95$ & 1 & 1 & 1 & 1 \\
\hline LASALLE $1 / 2$ & $12 / 94$ & 2 & 3 & 2 & 3 \\
\hline MONTICELLO & $08 / 96$ & 1 & 1 & 2 & 1 \\
\hline PALISADES & $07 / 95$ & 2 & 2 & 2 & 2 \\
\hline PERRY 1 & $02 / 95$ & 2 & 2 & 2 & 2 \\
\hline POINT BEACH $1 / 2$ & $06 / 96$ & 2 & 2 & 2 & 1 \\
\hline PRAIRIE ISLAND $1 / 2$ & $03 / 96$ & 1 & 1 & 2 & 1 \\
\hline QUAD CITIES $1 / 2$ & $08 / 95$ & 2 & 3 & 2 & 2 \\
\hline ZION $1 / 2$ & $02 / 96$ & 3 & 2 & 2 & 2 \\
\hline
\end{tabular}

NUREG-1214 
SALP SUMMARY

REGION 4

(REACTORS IN OPERATION)

\begin{tabular}{lccccc} 
PLANT NAME & RPT & OPS & MAINT & ENG & PS \\
\hline ARKANSAS 1/2 & $08 / 96$ & 1 & 2 & 1 & 1 \\
CALLAWAY & $06 / 95$ & 1 & 1 & 1 & 1 \\
COMANCHE PEAK 1/2 & $12 / 95$ & 1 & 1 & 2 & 1 \\
COOPER & $08 / 95$ & 2 & 3 & 3 & 2 \\
DIABLO CANYON 1/2 & $09 / 96$ & 2 & 2 & 2 & 1 \\
FORT CALHOUN & $03 / 96$ & 2 & 2 & 1 & 1 \\
GRAND GULF 1 & $03 / 96$ & 1 & 2 & 1 & 1 \\
PALO VERDE 1/2/3 & $07 / 96$ & 1 & 1 & 1 & 2 \\
RIVER BEND 1 & $08 / 95$ & 2 & 2 & 2 & 1 \\
SAN ONOFRE 2/3 & $02 / 96$ & 2 & 2 & 1 & 1 \\
SOUTH TEXAS 1/2 & $05 / 96$ & 2 & 1 & 2 & 1 \\
WASHINGTON NUCLEAR 2 & $09 / 95$ & 3 & 2 & 3 & 2 \\
WATERFORD 3 & $05 / 95$ & 2 & 1 & 1 & 1 \\
WOLF CREEK 1 & $11 / 95$ & 1 & 2 & 2 & 1
\end{tabular}

NUREG-1214 
Section 2

SALP History: Facilities in Operation

NUREG-1214 


\section{SALP HISTORY}

\section{(REACTORS IN OPERATION)}

PLANT NAME: BEAVER VALLEY

REGION: 1

\begin{tabular}{||c|c|c|c|c|c|c||}
\multicolumn{1}{c}{ UNIT } & RPT & ASSESSMENT PERIOD & OPS & MAINT & ENG & PS $^{1}$ \\
\hline $1 / 2$ & $07 / 95$ & $11 / 28 / 93-06 / 03 / 95$ & 2 & 1 & 2 & 1 \\
\hline $1 / 2$ & $02 / 94$ & $06 / 14 / 92-11 / 27 / 93$ & 1 & 2 & 2 & 1 \\
\hline $1 / 2$ & $10 / 92$ & $01 / 01 / 91-06 / 13 / 92$ & 1 & 2 & 2 & $1 / 1 / 1$ \\
\hline $1 / 2$ & $05 / 91$ & $09 / 01 / 89-12 / 31 / 90$ & 1 & 1 & 2 & $2 / 1 / 1$ \\
\hline $1 / 2$ & $02 / 90$ & $06 / 01 / 88-08 / 31 / 89$ & 2 & 2 & 2 & $2 / 1 / 1$ \\
\hline 1 & $11 / 88$ & $03 / 16 / 87-05 / 31 / 88$ & 1 & 2 & 2 & $2 / 1 / 1$ \\
\hline
\end{tabular}

PLANT NAME: CALVERT CLIFFS

REGION: 1

\begin{tabular}{|c|c|c|c|c|c|c|}
\hline UNIT & RPT & ASSESSMENT PERIOD & OPS & MAINT & ENG & $\mathrm{PS}^{1}$ \\
\hline $1 / 2$ & $07 / 95$ & $10 / 10 / 93-05 / 13 / 95$ & 1 & 2 & 1 & 2 \\
\hline $1 / 2$ & $11 / 93$ & $03 / 29 / 92-10 / 09 / 93$ & 1 & 2 & 1 & 2 \\
\hline $1 / 2$ & $08 / 92$ & $04 / 01 / 91-03 / 28 / 92$ & 1 & 2 & 2 & $1 / 2 / 1$ \\
\hline $1 / 2$ & $06 / 91$ & $01 / 01 / 90-03 / 31 / 91$ & 2 & 2 & 2 & $2 / 2 / 1$ \\
\hline $1 / 2$ & $06 / 90$ & $12 / 01 / 88-12 / 31 / 89$ & 3 & 3 & 2 & $2 / 2 / 1$ \\
\hline $1 / 2$ & $05 / 89$ & $09 / 01 / 87-11 / 30 / 88$ & 2 & 2 & 2 & $1 / 2 / 1$ \\
\hline
\end{tabular}

${ }^{1}$ SALP scores for radiological controls/emergency preparedness/security are listed for SALP evaluations conducted between June 1988 and July 1993. NUREG-1214 
SALP HISTORY

(REACTORS IN OPERATION)

PLANT NAME: FITZPATRICK

REGION: 1

\begin{tabular}{||c|c|c|c|c|c|c|}
\multicolumn{1}{l}{ UNIT } & RPT & ASSESSMENT PERIOD & OPS & MAINT & ENG & PS $^{1}$ \\
\hline & $12 / 95$ & $04 / 10 / 94-11 / 18 / 95$ & 2 & 2 & 2 & 2 \\
\hline \hline & $05 / 94$ & $04 / 18 / 93-04 / 09 / 94$ & 2 & 2 & 2 & 2 \\
\hline \hline & $09 / 93$ & $04 / 19 / 92-04 / 17 / 93$ & 2 & 2 & 3 & $2 / 1 / 1$ \\
\hline \hline & $08 / 92$ & $02 / 01 / 91-04 / 18 / 92$ & 3 & 3 & 3 & $2 / 1 / 1$ \\
\hline \hline & $07 / 91$ & $10 / 01 / 89-01 / 31 / 91$ & 2 & 2 & 2 & $3 / 1 / 1$ \\
\hline & $05 / 90$ & $05 / 01 / 88-09 / 30 / 89$ & 1 & 2 & 2 & $2 / 1 / 1$ \\
\hline
\end{tabular}

PLANT NAME: GINNA

REGION: 1

\begin{tabular}{||c|c|c|c|c|c|c||}
\multicolumn{1}{c}{ UNIT } & RPT & ASSESSMENT PERIOD & OPS & MAINT & ENG & PS $^{1}$ \\
\hline & $05 / 95$ & $09 / 12 / 93-03 / 11 / 95$ & 1 & 2 & 1 & 2 \\
\hline & $11 / 93$ & $01 / 19 / 92-09 / 11 / 93$ & 2 & 1 & 1 & 2 \\
\hline & $06 / 92$ & $10 / 01 / 90-01 / 18 / 92$ & 2 & 1 & 2 & $2 / 1 / 1$ \\
\hline & $12 / 90$ & $06 / 01 / 89-09 / 30 / 90$ & 2 & 2 & 2 & $2 / 1 / 2$ \\
\hline & $11 / 89$ & $12 / 01 / 87-05 / 31 / 89$ & 2 & 2 & 2 & $2 / 1 / 2$ \\
\hline
\end{tabular}

NUREG-1214 


\section{SALP HISTORY}

\section{(REACTORS IN OPERATION)}

PLANT NAME: HADDAM NECK ${ }^{2}$

REGION: 1

\begin{tabular}{||c|c|c|c|c|c|c||}
\multicolumn{1}{c}{ UNIT } & RPT & ASSESSMENT PERIOD & OPS & MAINT & ENG & PS $^{1}$ \\
\hline \hline & $11 / 94$ & $01 / 10 / 93-09 / 10 / 94$ & 2 & 2 & 2 & 1 \\
\hline \hline & $05 / 93$ & $07 / 14 / 91-01 / 09 / 93$ & 1 & 2 & 1 & $1 / 1 / 1$ \\
\hline \hline & $11 / 91$ & $02 / 16 / 90-07 / 13 / 91$ & 1 & 2 & 1 & $1 / 1 / 1$ \\
\hline \hline & $07 / 90$ & $08 / 01 / 88-02 / 15 / 90$ & 1 & 1 & 1 & $1 / 1 / 1$ \\
\hline & $12 / 88$ & $04 / 01 / 87-07 / 31 / 88$ & 1 & 2 & 1 & $1 / 1 / 1$ \\
\hline
\end{tabular}

PLANT NAME: HOPE CREEK

REGION: 1

\begin{tabular}{|c|c|c|c|c|c|c|}
\multicolumn{1}{c}{ UNIT } & RPT & ASSESSMENT PERIOD & OPS & MAINT & ENG & PS $^{1}$ \\
\hline 1 & $06 / 95$ & $06 / 20 / 93-04 / 22 / 95$ & 2 & 2 & 2 & 1 \\
\hline \hline 1 & $10 / 93$ & $12 / 29 / 91-06 / 19 / 93$ & 1 & 1 & 2 & $1 / 1 / 1$ \\
\hline 1 & $05 / 92$ & $08 / 01 / 90-12 / 28 / 91$ & 1 & 2 & 2 & $1 / 1 / 1$ \\
\hline \hline 1 & $11 / 90$ & $05 / 01 / 89-07 / 31 / 90$ & 1 & 2 & 1 & $1 / 1 / 1$ \\
\hline 1 & $09 / 89$ & $01 / 16 / 88-04 / 30 / 89$ & 1 & 2 & 2 & $1 / 2 / 1$ \\
\hline
\end{tabular}

${ }^{2}$ The current Haddam Neck SALP evaluation period has been extended untiT three months after the restart of the unit from the current refueling and configuration management plan review outage.

NUREG-1214 
(REACTORS IN OPERATION)

PLANT NAME: INDIAN POINT REGION: 1

\begin{tabular}{|c|c|c|c|c|c|c|}
\multicolumn{1}{c}{ UNIT } & RPT & ASSESSMENT PERIOD & OPS & MAINT & ENG & PS $^{1}$ \\
\hline 2 & $10 / 95$ & $03 / 20 / 94-09 / 16 / 95$ & 2 & 1 & 1 & 2 \\
\hline 2 & $05 / 94$ & $09 / 27 / 92-03 / 19 / 94$ & 1 & 1 & 1 & 2 \\
\hline 2 & $01 / 93$ & $05 / 01 / 91-09 / 26 / 92$ & 1 & 2 & 1 & $1 / 2 / 1$ \\
\hline 2 & $09 / 91$ & $04 / 01 / 90-04 / 30 / 91$ & 1 & 2 & 2 & $2 / 2 / 1$ \\
\hline 2 & $08 / 90$ & $03 / 01 / 89-03 / 31 / 90$ & 1 & 2 & 2 & $2 / 2 / 1$ \\
\hline 2 & $06 / 89$ & $02 / 08 / 88-02 / 28 / 89$ & 2 & 2 & 3 & $2 / 2 / 1$ \\
\hline
\end{tabular}

PLANT NAME: INDIAN POINT REGION: 1

\begin{tabular}{|c|c|c|c|c|c|c|}
\hline UNIT & RPT & ASSESSMENT PERIOD & OPS & MAINT & ENG & $\mathrm{PS}^{1}$ \\
\hline 3 & $04 / 96$ & $08 / 16 / 92-03 / 02 / 96$ & 3 & 2 & 3 & 1 \\
\hline 3 & $12 / 92$ & $06 / 02 / 91-08 / 15 / 92$ & 2 & 2 & 3 & $1 / 2 / 2$ \\
\hline 3 & $10 / 91$ & $12 / 01 / 89-06 / 01 / 91$ & 2 & 2 & 2 & $1 / 1 / 2$ \\
\hline 3 & $07 / 90$ & $09 / 01 / 88-11 / 30 / 89$ & 2 & 2 & 2 & $1 / 1 / 3$ \\
\hline 3 & $01 / 89$ & $06 / 01 / 87-08 / 31 / 88$ & 2 & 2 & 3 & $1 / 1 / 1$ \\
\hline
\end{tabular}


(REACTORS IN OPERATION)

PLANT NAME: LIMERICK

REGION: 1

\begin{tabular}{|c|c|c|c|c|c|c|}
\hline UNIT & RPT & ASSESSMENT PERIOD & OPS & MAINT & ENG & $\mathrm{PS}^{1}$ \\
\hline $1 / 2$ & $05 / 95$ & $09 / 26 / 93-04 / 01 / 95$ & 1 & 1 & 1 & 1 \\
\hline $1 / 2$ & $11 / 93$ & $03 / 15 / 92-09 / 25 / 93$ & 1 & 2 & 1 & 2 \\
\hline $1 / 2$ & $08 / 92$ & $10 / 16 / 90-03 / 14 / 92$ & 1 & 2 & 1 & $1 / 1 / 1$ \\
\hline $1 / 2$ & $02 / 91$ & $09 / 01 / 89-10 / 15 / 90$ & 1 & 1 & 2 & $1 / 2 / 1$ \\
\hline 1 & $02 / 90$ & $05 / 01 / 88-08 / 31 / 89$ & 1 & 1 & 2 & $1 / 3 / 1$ \\
\hline 1 & $08 / 88$ & $02 / 01 / 87-04 / 30 / 88$ & 1 & 1 & 2 & $1 / 2 / 1$ \\
\hline
\end{tabular}

PLANT NAME: MAINE YANKEE

REGION: 1

\begin{tabular}{|c|c|c|c|c|c|c|}
\multicolumn{1}{c}{ UNIT } & RPT & ASSESSMENT PERIOD & OPS & \multicolumn{1}{c}{ MAINT } & ENG & PS $^{1}$ \\
\hline \hline & $10 / 95$ & $02 / 06 / 94-08 / 26 / 95$ & 1 & 2 & 1 & 2 \\
\hline \hline & $03 / 94$ & $06 / 28 / 92-02 / 05 / 94$ & 1 & 2 & 2 & 1 \\
\hline \hline & $11 / 92$ & $03 / 01 / 91-06 / 27 / 92$ & 1 & 2 & 2 & $2 / 2 / 1$ \\
\hline \hline & $06 / 91$ & $11 / 01 / 89-02 / 28 / 91$ & 1 & 1 & 2 & $2 / 2 / 2$ \\
\hline \hline & $05 / 90$ & $08 / 01 / 88-10 / 31 / 89$ & 1 & 1 & 2 & $2 / 2 / 2$ \\
\hline \hline & $01 / 89$ & $02 / 01 / 87-07 / 31 / 88$ & 1 & 1 & 2 & $3 / 1 / 2$ \\
\hline
\end{tabular}


(REACTORS IN OPERATION)

PLANT NAME: MILLSTONE ${ }^{3}$

REGION: 1

\begin{tabular}{|c|c|c|c|c|c|c|}
\hline UNIT & RPT & ASSESSMENT PERIOD & OPS & MAINT & ENG & $\mathrm{PS}^{1}$ \\
\hline $1 / 3$ & $08 / 94$ & $04 / 04 / 93-07 / 09 / 94$ & 2 & 2 & 2 & 2 \\
\hline $1 / 3$ & $10 / 93$ & $02 / 16 / 92-04 / 03 / 93$ & 2 & 2 & 2 & $1 / 2 / 2$ \\
\hline $1 / 3$ & $08 / 92$ & $12 / 16 / 90-02 / 15 / 92$ & 2 & 2 & 2 & $2 / 2 / 2$ \\
\hline 1 & $05 / 91$ & $06 / 16 / 89-12 / 15 / 90$ & 1 & 1 & 2 & $2 / 1 / 1$ \\
\hline 3 & $05 / 91$ & $10 / 16 / 89-12 / 15 / 90$ & 2 & 1 & 2 & $2 / 1 / 1$ \\
\hline 1 & $04 / 90$ & $01 / 01 / 88-06 / 15 / 89$ & 1 & 1 & 2 & $2 / 1 / 1$ \\
\hline 3 & $04 / 90$ & $06 / 01 / 88-10 / 15 / 89$ & 2 & 1 & 2 & $1 / 1 / 1$ \\
\hline
\end{tabular}

PLANT NAME: MILLSTONE ${ }^{3}$

REGION: 1

\begin{tabular}{|c|c|c|c|c|c|c|}
\hline UNIT & RPT & ASSESSMENT PERIOD & OPS & MAINT & ENG & $\mathrm{PS}^{1}$ \\
\hline 2 & $08 / 94$ & $04 / 04 / 93-07 / 09 / 94$ & 3 & 3 & 2 & 2 \\
\hline 2 & $10 / 93$ & $02 / 16 / 92-04 / 03 / 93$ & 2 & 2 & 2 & $1 / 2 / 2$ \\
\hline 2 & $08 / 92$ & $12 / 16 / 90-02 / 15 / 92$ & 2 & 2 & 2 & $2 / 2 / 2$ \\
\hline 2 & $05 / 91$ & $06 / 16 / 89-12 / 15 / 90$ & 1 & 2 & 2 & $2 / 1 / 1$ \\
\hline 2 & $04 / 90$ & $01 / 01 / 88-06 / 15 / 89$ & 1. & 2 & 1 & $1 / 1 / 1$ \\
\hline
\end{tabular}

${ }^{3}$ The Millstone SALP evaluations are currently superseded by other NRC evaluation activities associated with 10 CFR $50.54 \mathrm{f}$ letters. A11 three units require Commission approval to restart.

NUREG-1214 


\section{SALP HISTORY}

\section{(REACTORS IN OPERATION)}

PLANT NAME: NINE MILE POINT REGION: 1

\begin{tabular}{||c|c|c|c|c|c|c||}
\multicolumn{1}{c}{ UNIT } & RPT & \multicolumn{1}{c}{ ASSESSMENT PERIOD } & OPS & \multicolumn{1}{c|}{ MAINT } & ENG & PS $^{1}$ \\
\hline $1 / 2$ & $07 / 96$ & $01 / 29 / 95-06 / 01 / 96$ & 1 & 2 & 2 & 2 \\
\hline \hline $1 / 2$ & $03 / 95$ & $08 / 15 / 93-01 / 28 / 95$ & 1 & 2 & 2 & 2 \\
\hline \hline $1 / 2$ & $09 / 93$ & $05 / 24 / 92-08 / 14 / 93$ & 2 & 2 & 1 & 2 \\
\hline \hline $1 / 2$ & $10 / 92$ & $04 / 01 / 91-05 / 23 / 92$ & 2 & 2 & 2 & $2 / 1 / 1$ \\
\hline \hline $1 / 2$ & $08 / 91$ & $03 / 01 / 90-03 / 31 / 91$ & 2 & 2 & 2 & $2 / 1 / 1$ \\
\hline \hline $1 / 2$ & $08 / 90$ & $03 / 01 / 89-02 / 28 / 90$ & 3 & 3 & 2 & $2 / 1 / 1$ \\
\hline \hline $1 / 2$ & $07 / 89$ & $03 / 01 / 88-02 / 28 / 89$ & 3 & 3 & 3 & $2 / 1 / 1$ \\
\hline
\end{tabular}

PLANT NAME: OYSTER CREEK REGION: 1

\begin{tabular}{||c|c|c|c|c|c|c||}
\multicolumn{1}{c}{ UNIT } & RPT & ASSESSMENT PERIOD & OPS & MAINT & ENG & PS $^{1}$ \\
\hline \hline & $08 / 95$ & $12 / 12 / 93-06 / 24 / 95$ & 1 & 2 & 1 & 2 \\
\hline \hline & $01 / 94$ & $07 / 19 / 92-12 / 11 / 93$ & 2 & 2 & 1 & 2 \\
\hline \hline & $11 / 92$ & $04 / 16 / 91-07 / 18 / 92$ & 2 & 2 & 2 & $2 / 1 / 2$ \\
\hline \hline & $08 / 91$ & $03 / 16 / 90-04 / 15 / 91$ & 2 & 2 & 2 & $2 / 1 / 2$ \\
\hline \hline & $09 / 90$ & $02 / 01 / 89-03 / 15 / 90$ & 2 & 2 & 2 & $3 / 2 / 2$ \\
\hline \hline & $08 / 89$ & $10 / 01 / 87-01 / 31 / 89$ & 3 & 2 & 2 & $3 / 2 / 2$ \\
\hline
\end{tabular}




\section{SALP HISTORY}

\section{(REACTORS IN OPERATION)}

PLANT NAME: PEACH BOTTOM

REGION: 1

\begin{tabular}{|c|c|c|c|c|c|c||}
\multicolumn{1}{c}{ UNIT } & RPT & ASSESSMENT PERIOD & OPS & MAINT & ENG & PS $^{1}$ \\
\hline $2 / 3$ & $12 / 95$ & $05 / 01 / 94-10 / 14 / 95$ & 1 & 1 & 2 & 1 \\
\hline $2 / 3$ & $06 / 94$ & $11 / 01 / 92-04 / 30 / 94$ & 1 & 2 & 2 & 2 \\
\hline $2 / 3$ & $03 / 93$ & $08 / 04 / 91-10 / 31 / 92$ & 2 & 2 & 2 & $2 / 1 / 1$ \\
\hline $2 / 3$ & $12 / 91$ & $06 / 01 / 90-08 / 03 / 91$ & 2 & 2 & 2 & $2 / 1 / 1$ \\
\hline $2 / 3$ & $10 / 90$ & $07 / 01 / 89-05 / 31 / 90$ & 2 & 2 & 2 & $2 / 1 / 1$ \\
\hline \hline $2 / 3$ & $02 / 90$ & $08 / 01 / 88-06 / 30 / 89$ & 2 & 2 & 2 & $2 / 1 / 2$ \\
\hline $2 / 3$ & $12 / 88$ & $06 / 01 / 87-07 / 31 / 88$ & 2 & 2 & 1 & $2 / 2 / 3$ \\
\hline
\end{tabular}

PLANT NAME: PILGRIM

REGION: 1

\begin{tabular}{l} 
UNIT \\
\hline \begin{tabular}{|c|c|c|c|c|c|c||}
\hline 1 & RPT & ASSESSMENT PERIOD & OPS & MAINT & ENG & PS $^{1}$ \\
\hline \hline 1 & $05 / 96$ & $10 / 09 / 94-04 / 06 / 96$ & 2 & 2 & 1 & 2 \\
\hline \hline 1 & $11 / 94$ & $03 / 14 / 93-10 / 08 / 94$ & 1 & 2 & 1 & 1 \\
\hline \hline 1 & $08 / 93$ & $09 / 29 / 91-03 / 13 / 93$ & 1 & 2 & 2 & $1 / 1 / 1$ \\
\hline \hline 1 & $11 / 90$ & $07 / 01 / 89-08 / 15 / 90$ & 2 & 2 & 1 & $1 / 2 / 1$ \\
\hline \hline 1 & $12 / 89$ & $05 / 16 / 88-06 / 30 / 89$ & 2 & 2 & 1 & $2 / 2 / 1$ \\
\hline
\end{tabular}
\end{tabular}

NUREG-1214 


\section{SALP HISTORY}

(REACTORS IN OPERATION)

PLANT NAME: SALEM ${ }^{4}$

REGION:

\begin{tabular}{|c|c|c|c|c|c|c|}
\multicolumn{1}{c}{ UNIT } & RPT & ASSESSMENT PERIOD & OPS & MAINT & ENG & PS $^{1}$ \\
\hline $1 / 2$ & $01 / 95$ & $06 / 20 / 93-11 / 05 / 94$ & 3 & 3 & 2 & 1 \\
\hline \hline $1 / 2$ & $10 / 93$ & $12 / 29 / 91-06 / 19 / 93$ & 2 & 2 & 2 & $1 / 1 / 1$ \\
\hline \hline $1 / 2$ & $05 / 92$ & $08 / 01 / 90-12 / 28 / 91$ & 2 & 2 & 2 & $2 / 1 / 1$ \\
\hline \hline $1 / 2$ & $11 / 90$ & $05 / 01 / 89-07 / 31 / 90$ & 2 & 2 & 2 & $2 / 1 / 1$ \\
\hline \hline $1 / 2$ & $09 / 89$ & $01 / 01 / 88-04 / 30 / 89$ & 3 & 2 & 2 & $2 / 2 / 1$ \\
\hline
\end{tabular}

PLANT NAME: SEABROOK

REGION: 1

\begin{tabular}{|c|c|c|c|c|c|c|}
\hline UNIT & RPT & ASSESSMENT PERIOD & OPS & MAINT & ENG & $\mathrm{PS}^{1}$ \\
\hline 1 & $06 / 96$ & $01 / 08 / 95-05 / 04 / 96$ & 1 & 2 & 1 & 1 \\
\hline 1 & $02 / 95$ & $08 / 29 / 93-01 / 07 / 95$ & 2 & 2 & 1 & 1 \\
\hline 1 & $11 / 93$ & $03 / 01 / 92-08 / 28 / 93$ & 2 & 2 & 1 & 1 \\
\hline 1 & $07 / 92$ & $11 / 01 / 90-02 / 29 / 92$ & 2 & 2 & 2 & $2 / 1 / 1$ \\
\hline 1 & $01 / 91$ & $07 / 01 / 89-10 / 31 / 90$ & 2 & 2 & 2 & $2 / 1 / 1$ \\
\hline
\end{tabular}

${ }^{4}$ The current Salem SALP evaluation period has been extended until one year after the restart of the first unit from its current outage.

NUREG-1214

2-9 


\section{SALP HISTORY}

\section{(REACTORS IN OPERATION)}

PLANT NAME: SUSQUEHANNA

REGION: 1

\begin{tabular}{|c|c|c|c|c|c|c|}
\hline UNIT & RPT & ASSESSMENT PERIOD & OPS & MAINT & ENG & $\mathrm{PS}^{1}$ \\
\hline $1 / 2$ & $09 / 95$ & $02 / 27 / 94-08 / 05 / 95$ & 1 & 1 & 1 & 1 \\
\hline $1 / 2$ & $04 / 94$ & $04 / 19 / 92-02 / 26 / 94$ & 2 & 1 & 1 & 1 \\
\hline $1 / 2$ & $08 / 92$ & $12 / 01 / 90-04 / 18 / 92$ & 1 & 1 & 1 & $2 / 1 / 1$ \\
\hline $1 / 2$ & $03 / 91$ & $08 / 01 / 89-11 / 30 / 90$ & 1 & 1 & 2 & $2 / 1 / 1$ \\
\hline $1 / 2$ & $01 / 90$ & $02 / 01 / 88-07 / 31 / 89$ & 1 & 1 & 1 & $2 / 2 / 1$ \\
\hline
\end{tabular}

PLANT NAME: THREE MILE ISLAND REGION: 1

\begin{tabular}{|c|c|c|c|c|c|c||}
\multicolumn{1}{c}{ UNIT } & RPT & \multicolumn{1}{c}{ ASSESSMENT PERIOD } & OPS & \multicolumn{1}{c}{ MAINT } & ENG & PS $^{1}$ \\
\hline \hline 1 & $09 / 96$ & $02 / 19 / 95-08 / 03 / 96$ & 1 & 1 & 2 & 2 \\
\hline \hline 1 & $03 / 95$ & $08 / 01 / 93-02 / 18 / 95$ & 2 & 1 & 1 & 1 \\
\hline \hline 1 & $10 / 93$ & $11 / 17 / 91-07 / 31 / 93$ & 2 & 1 & 1 & 1 \\
\hline \hline 1 & $03 / 92$ & $05 / 16 / 90-11 / 16 / 91$ & 1 & 2 & 1 & $1 / 1 / 1$ \\
\hline 1 & $10 / 90$ & $01 / 16 / 89-05 / 15 / 90$ & 1 & 1 & 1 & $1 / 1 / 1$ \\
\hline \hline 1 & $06 / 89$ & $11 / 01 / 87-01 / 15 / 89$ & 1 & 2 & 2 & $1 / 1 / 1$ \\
\hline
\end{tabular}




\section{SALP HISTORY}

(REACTORS IN OPERATION)

PLANT NAME: VERMONT YANKEE

REGION: 1

\begin{tabular}{|c|c|c|c|c|c|c||}
\multicolumn{1}{l}{ UNIT } & RPT & ASSESSMENT PERIOD & OPS & MAINT & ENG & PS $^{1}$ \\
\hline \hline & $08 / 95$ & $01 / 16 / 94-07 / 15 / 95$ & 2 & 2 & 2 & 1 \\
\hline \hline & $02 / 94$ & $08 / 02 / 92-01 / 15 / 94$ & 2 & 2 & 2 & 1 \\
\hline \hline & $01 / 93$ & $03 / 17 / 91-08 / 01 / 92$ & 1 & 1 & 2 & $2 / 1 / 3$ \\
\hline \hline & $07 / 91$ & $10 / 01 / 89-03 / 16 / 91$ & 1 & 1 & 1 & $2 / 1 / 2$ \\
\hline & $03 / 90$ & $07 / 01 / 88-09 / 30 / 89$ & 1 & 1 & 1 & $2 / 1 / 2$ \\
\hline
\end{tabular}




\section{SALP HISTORY}

\section{(REACTORS IN OPERATION)}

PLANT NAME: BROWNS FERRY 5

REGION: 2

\begin{tabular}{|c|c|c|c|c|c|c||}
\multicolumn{1}{c}{ UNIT } & RPT & ASSESSMENT PERIOD & OPS & MAINT & ENG & PS $^{1}$ \\
\hline 2 & $04 / 95$ & $09 / 19 / 93-03 / 18 / 95$ & 2 & 2 & 2 & 1 \\
\hline 2 & $11 / 93$ & $05 / 24 / 92-09 / 18 / 93$ & 1 & 2 & 2 & 1 \\
\hline \hline 2 & $09 / 92$ & $05 / 24 / 91-05 / 23 / 92$ & 1 & 2 & 2 & $1 / 1 / 2$ \\
\hline \hline 2 & $08 / 90$ & $01 / 03 / 89-03 / 31 / 90$ & 2 & 3 & 2 & $1 / 2 / 2$ \\
\hline
\end{tabular}

PLANT NAME: BRUNSWICK

REGION: 2

\begin{tabular}{||c|c|c|c|c|c|c||}
\multicolumn{1}{c}{ UNIT } & RPT & ASSESSMENT PERIOD & OPS & MAINT & ENG & PS $^{1}$ \\
\hline \hline $1 / 2$ & $06 / 95$ & $11 / 07 / 93-05 / 13 / 95$ & 1 & 1 & 1 & 1 \\
\hline \hline $1 / 2$ & $12 / 93$ & $11 / 01 / 92-11 / 06 / 93$ & 1 & 2 & 2 & 1 \\
\hline \hline $1 / 2$ & $02 / 93$ & $11 / 03 / 91-10 / 31 / 92$ & 2 & 3 & 2 & $2 / 2 / 2$ \\
\hline \hline $1 / 2$ & $03 / 92$ & $10 / 01 / 90-11 / 02 / 91$ & 2 & 3 & 2 & $2 / 2 / 2$ \\
\hline \hline $1 / 2$ & $01 / 91$ & $09 / 01 / 89-09 / 30 / 90$ & 2 & 2 & 3 & $2 / 2 / 1$ \\
\hline \hline $1 / 2$ & $12 / 89$ & $09 / 01 / 88-08 / 31 / 89$ & 2 & 2 & 2 & $2 / 2 / 1$ \\
\hline \hline $1 / 2$ & $12 / 88$ & $07 / 01 / 87-08 / 31 / 88$ & 2 & 2 & 3 & $2 / 2 / 1$ \\
\hline
\end{tabular}

${ }^{5}$ Browns Ferry Unit 3 restarted from an extended shutdown during 1995. The next SALP evaluation for Browns Ferry will cover both Units 2 and 3 . NUREG-1214 


\section{SALP HISTORY}

\section{(REACTORS IN OPERATION)}

PLANT NAME: CATAWBA

REGION: 2

\begin{tabular}{|c|c|c|c|c|c|c||}
\multicolumn{1}{c}{ UNIT } & RPT & ASSESSMENT PERIOD & OPS & MAINT & ENG & PS $^{1}$ \\
\hline $1 / 2$ & $11 / 95$ & $10 / 03 / 93-10 / 07 / 95$ & 2 & 2 & 2 & 2 \\
\hline \hline $1 / 2$ & $11 / 93$ & $05 / 03 / 92-10 / 02 / 93$ & 2 & 2 & 2 & 1 \\
\hline $1 / 2$ & $08 / 92$ & $02 / 03 / 91-05 / 02 / 92$ & 2 & 2 & 2 & $1 / 1 / 1$ \\
\hline \hline $1 / 2$ & $05 / 91$ & $11 / 01 / 89-02 / 02 / 91$ & 2 & 2 & 2 & $2 / 1 / 1$ \\
\hline \hline $1 / 2$ & $03 / 90$ & $08 / 01 / 88-10 / 31 / 89$ & 2 & 2 & 2 & $2 / 1 / 2$ \\
\hline \hline $1 / 2$ & $12 / 88$ & $08 / 01 / 87-07 / 31 / 88$ & 2 & 2 & 2 & $2 / 2 / 2$ \\
\hline
\end{tabular}

PLANT NAME: CRYSTAL RIVER REGION: 2

\begin{tabular}{|c|c|c|c|c|c|c||}
\multicolumn{1}{c}{ UNIT } & RPT & ASSESSMENT PERIOD & OPS & MAINT & ENG & PS $^{1}$ \\
\hline 3 & $10 / 95$ & $02 / 20 / 94-09 / 16 / 95$ & 2 & 2 & 2 & 1 \\
\hline \hline 3 & $04 / 94$ & $08 / 23 / 92-02 / 19 / 94$ & 2 & 2 & 1 & 1 \\
\hline \hline 3 & $01 / 93$ & $04 / 28 / 91-08 / 22 / 92$ & 2 & 2 & 2 & $1 / 1 / 1$ \\
\hline \hline 3 & $07 / 91$ & $03 / 01 / 90-04 / 27 / 91$ & 1 & 2 & 2 & $1 / 1 / 1$ \\
\hline \hline 3 & $06 / 90$ & $01 / 01 / 89-02 / 28 / 90$ & 2 & 2 & 2 & $1 / 1 / 1$ \\
\hline 3 & $05 / 89$ & $09 / 01 / 87-12 / 31 / 88$ & 2 & 2 & 3 & $2 / 2 / 1$ \\
\hline
\end{tabular}

NUREG-1214 


\section{SALP HISTORY}

\section{(REACTORS IN OPERATION)}

PLANT NAME: FARLEY

REGION: 2

\begin{tabular}{|c|c|c|c|c|c|c|}
\multicolumn{1}{c}{ UNIT } & RPT & ASSESSMENT PERIOD & OPS & MAINT & ENG & PS $^{1}$ \\
\hline $1 / 2$ & $05 / 95$ & $09 / 26 / 93-03 / 25 / 95$ & 1 & 2 & 1 & 1 \\
\hline \hline $1 / 2$ & $11 / 93$ & $05 / 31 / 92-09 / 25 / 93$ & 1 & 2 & 1 & 1 \\
\hline $1 / 2$ & $09 / 92$ & $01 / 01 / 91-05 / 30 / 92$ & 2 & 2 & 2 & $1 / 2 / 1$ \\
\hline \hline $1 / 2$ & $05 / 91$ & $08 / 01 / 89-12 / 31 / 90$ & 1 & 1 & 2 & $1 / 2 / 2$ \\
\hline $1 / 2$ & $12 / 89$ & $04 / 01 / 88-07 / 31 / 89$ & 1 & 1 & 2 & $1 / 2 / 1$ \\
\hline
\end{tabular}

PLANT NAME: HARRIS

REGION: 2

\begin{tabular}{|c|c|c|c|c|c|c|}
\multicolumn{1}{c}{ UNIT } & RPT & ASSESSMENT PERIOD & OPS & MAINT & ENG & PS $^{1}$ \\
\hline \hline 1 & $04 / 96$ & $04 / 03 / 94-03 / 16 / 96$ & 1 & 1 & 1 & 1 \\
\hline \hline 1 & $05 / 94$ & $09 / 27 / 92-04 / 02 / 94$ & 1 & 1 & 1 & 1 \\
\hline \hline 1 & $01 / 93$ & $06 / 02 / 91-09 / 26 / 92$ & 1 & 2 & 1 & $1 / 1 / 1$ \\
\hline \hline 1 & $09 / 91$ & $12 / 01 / 89-06 / 01 / 91$ & 1 & 2 & 1 & $1 / 1 / 1$ \\
\hline \hline 1 & $04 / 90$ & $07 / 01 / 88-11 / 30 / 89$ & 1 & 2 & 1 & $1 / 1 / 2$ \\
\hline
\end{tabular}




\section{SALP HISTORY}

\section{(REACTORS IN OPERATION)}

PLANT NAME: HATCH

REGION: 2

\begin{tabular}{l}
\multicolumn{1}{c}{ UNIT } \\
\hline \begin{tabular}{|c|c|c|c|c|c|c|}
\hline $1 / 2$ & RPT & ASSESSMENT PERIOD & OPS & MAINT & ENG & PS $^{1}$ \\
\hline \hline $1 / 2$ & $01 / 94$ & $11 / 28 / 93-05 / 27 / 95$ & 2 & 2 & 1 & 2 \\
\hline $1 / 2$ & $09 / 92$ & $05 / 31 / 92-11 / 27 / 93$ & 2 & 2 & 1 & 1 \\
\hline \hline $1 / 2$ & $07 / 91$ & $10 / 01 / 89-03 / 02 / 91$ & 1 & 2 & 2 & $2 / 1 / 2$ \\
\hline \hline $1 / 2$ & $01 / 90$ & $07 / 01 / 88-09 / 30 / 89$ & 1 & 1 & 2 & $2 / 1 / 2$ \\
\hline
\end{tabular}
\end{tabular}

PLANT NAME: MCGUIRE REGION: 2

\begin{tabular}{|c|c|c|c|c|c|c|}
\multicolumn{1}{c}{ UNIT } & RPT & ASSESSMENT PERIOD & OPS & MAINT & ENG & PS $^{1}$ \\
\hline \hline $1 / 2$ & $09 / 95$ & $02 / 06 / 94-08 / 12 / 95$ & 2 & 2 & 1 & 1 \\
\hline \hline $1 / 2$ & $03 / 94$ & $08 / 02 / 92-02 / 05 / 94$ & 2 & 3 & 2 & 2 \\
\hline \hline $1 / 2$ & $11 / 92$ & $02 / 03 / 91-08 / 01 / 92$ & 2 & 2 & 1 & $1 / 1 / 1$ \\
\hline \hline $1 / 2$ & $05 / 91$ & $11 / 01 / 89-02 / 02 / 91$ & 2 & 2 & 1 & $1 / 1 / 1$ \\
\hline \hline $1 / 2$ & $03 / 90$ & $08 / 01 / 88-10 / 31 / 89$ & 2 & 2 & 2 & $1 / 1 / 2$ \\
\hline \hline $1 / 2$ & $12 / 88$ & $08 / 01 / 87-07 / 31 / 88$ & 2 & 2 & 2 & $2 / 1 / 2$ \\
\hline
\end{tabular}




\section{SALP HISTORY}

\section{(REACTORS IN OPERATION)}

PLANT NAME: NORTH ANNA

REGION:

\begin{tabular}{|c|c|c|c|c|c|c|}
\hline UNIT & RPT & ASSESSMENT PERIOD & OPS & MAINT & ENG & $\mathrm{PS}^{1}$ \\
\hline $1 / 2$ & $01 / 95$ & $04 / 04 / 93-12 / 24 / 94$ & 1 & 1 & 1 & 1 \\
\hline $1 / 2$ & $08 / 93$ & $11 / 03 / 91-04 / 03 / 93$ & 1 & 1 & 1 & $1 / 1 / 1$ \\
\hline $1 / 2$ & $02 / 92$ & $09 / 01 / 90-11 / 02 / 91$ & 1 & 2 & 2 & $1 / 1 / 1$ \\
\hline $1 / 2$ & $12 / 90$ & $06 / 01 / 89-08 / 31 / 90$ & 1 & 2 & 1 & $2 / 1 / 2$ \\
\hline $1 / 2$ & $10 / 89$ & $05 / 01 / 88-05 / 31 / 89$ & 1 & 2 & 2 & $2 / 2 / 2$ \\
\hline
\end{tabular}

PLANT NAME: OCONEE

REGION: 2

UNIT
\begin{tabular}{|c|c|c|c|c|c|c||}
\hline RPT & ASSESSMENT PERIOD & OPS & MAINT & ENG & PS $^{1}$ \\
\hline $1 / 2 / 3$ & $06 / 96$ & $10 / 30 / 94-05 / 04 / 96$ & 1 & 2 & 1 & 1 \\
\hline $1 / 2 / 3$ & $12 / 94$ & $05 / 02 / 93-10 / 29 / 94$ & 2 & 2 & 2 & 1 \\
\hline $1 / 2 / 3$ & $09 / 93$ & $02 / 02 / 92-05 / 01 / 93$ & 2 & 2 & 2 & $1 / 1 / 1$ \\
\hline $1 / 2 / 3$ & $06 / 92$ & $08 / 01 / 90-02 / 01 / 92$ & 2 & 2 & 2 & $1 / 1 / 1$ \\
\hline $1 / 2 / 3$ & $11 / 90$ & $02 / 01 / 89-07 / 31 / 90$ & 1 & 2 & 1 & $1 / 1 / 2$ \\
\hline $1 / 2 / 3$ & $06 / 89$ & $08 / 01 / 87-01 / 31 / 89$ & 1 & 2 & 2 & $1 / 1 / 2$ \\
\hline
\end{tabular}

NUREG-1214 


\section{SALP HISTORY}

\section{(REACTORS IN OPERATION)}

PLANT NAME: ROBINSON

REGION:

\begin{tabular}{||c|c|c|c|c|c|c|}
\multicolumn{1}{c}{ UNIT } & RPT & ASSESSMENT PERIOD & OPS & MAINT & ENG & PS $^{1}$ \\
\hline 2 & $07 / 95$ & $12 / 26 / 93-06 / 17 / 95$ & 2 & 2 & 2 & 2 \\
\hline 2 & $02 / 94$ & $06 / 28 / 92-12 / 25 / 93$ & 2 & 3 & 2 & 2 \\
\hline 2 & $10 / 92$ & $03 / 31 / 91-06 / 27 / 92$ & 1 & 2 & 2 & $1 / 2 / 1$ \\
\hline 2 & $07 / 91$ & $01 / 01 / 90-03 / 30 / 91$ & 2 & 2 & 2 & $1 / 2 / 1$ \\
\hline 2 & $05 / 90$ & $11 / 01 / 88-12 / 31 / 89$ & 2 & 2 & 2 & $1 / 2 / 1$ \\
\hline 2 & $02 / 89$ & $07 / 01 / 87-10 / 31 / 88$ & 2 & 2 & 2 & $2 / 2 / 1$ \\
\hline
\end{tabular}

PLANT NAME: SEQUOYAH

REGION: 2

\begin{tabular}{l|c|c|c|c|c|c|c|} 
UNIT & RPT & ASSESSMENT PERIOD & OPS & MAINT & ENG & PS $^{1}$ \\
\hline $1 / 2$ & $09 / 96$ & $01 / 08 / 95-07 / 27 / 96$ & 2 & 2 & 2 & 2 \\
\hline \hline $1 / 2$ & $02 / 95$ & $10 / 10 / 93-01 / 07 / 95$ & 2 & 2 & 2 & 2 \\
\hline \hline $1 / 2$ & $12 / 93$ & $08 / 02 / 92-10 / 09 / 93$ & 3 & 3 & 2 & 1 \\
\hline $1 / 2$ & $11 / 92$ & $06 / 02 / 91-08 / 01 / 92$ & 2 & 2 & 2 & $2 / 1 / 1$ \\
\hline \hline $1 / 2$ & $10 / 91$ & $03 / 06 / 90-06 / 01 / 91$ & 2 & 2 & 2 & $2 / 1 / 1$ \\
\hline $1 / 2$ & $07 / 90$ & $02 / 04 / 89-03 / 05 / 90$ & 2 & 2 & 2 & $2 / 2 / 2$ \\
\hline $1 / 2$ & $06 / 89$ & $02 / 04 / 88-02 / 03 / 89$ & 2 & 2 & 3 & $2 / 2 / 2$ \\
\hline
\end{tabular}




\section{SALP HISTORY}

\section{(REACTORS IN OPERATION)}

PLANT NAME: ST LUCIE

REGION: 2

\begin{tabular}{l} 
UNIT \\
\hline \begin{tabular}{|c|c|c|c|c|c|c||}
\hline $1 / 2$ & RPT & ASSESSMENT PERIOD & OPS & MAINT & ENG & PS $^{1}$ \\
\hline \hline $1 / 2$ & $02 / 96$ & $01 / 02 / 94-01 / 06 / 96$ & 2 & 2 & 1 & 1 \\
\hline $1 / 2$ & $07 / 92$ & $11 / 01 / 90-05 / 02 / 92$ & 1 & 1 & 1 & $1 / 1 / 1$ \\
\hline \hline $1 / 2$ & $01 / 91$ & $05 / 01 / 89-10 / 31 / 90$ & 1 & 2 & 1 & $1 / 1 / 1$ \\
\hline \hline $1 / 2$ & $09 / 89$ & $11 / 01 / 87-04 / 30 / 89$ & 1 & 1 & 1 & $2 / 1 / 2$ \\
\hline
\end{tabular}
\end{tabular}

PLANT NAME: SUMMER

REGION: 2

\begin{tabular}{l|c|c|c|c|c|c|c||} 
UNIT & RPT & ASSESSMENT PERIOD & OPS & MAINT & ENG & PS $^{1}$ \\
\hline & $03 / 95$ & $02 / 28 / 93-01 / 28 / 95$ & 1 & 1 & 2 & 1 \\
\hline \hline & $06 / 93$ & $09 / 01 / 91-02 / 27 / 93$ & 1 & 1 & 2 & $1 / 2 / 1$ \\
\hline \hline & $01 / 92$ & $05 / 01 / 90-08 / 31 / 91$ & 1 & 1 & 2 & $1 / 2 / 1$ \\
\hline \hline & $08 / 90$ & $01 / 01 / 89-04 / 30 / 90$ & 1 & 1 & 2 & $1 / 2 / 1$ \\
\hline \hline & $05 / 89$ & $08 / 01 / 87-12 / 31 / 88$ & 2 & 1 & 2 & $1 / 2 / 2$ \\
\hline
\end{tabular}




\section{SALP HISTORY}

(REACTORS IN OPERATION)

PLANT NAME: SURRY

REGION: 2

\begin{tabular}{|c|c|c|c|c|c|c|}
\multicolumn{1}{c}{ UNIT } & RPT & ASSESSMENT PERIOD & OPS & MAINT & ENG & PS $^{1}$ \\
\hline $1 / 2$ & $02 / 95$ & $07 / 04 / 93-01 / 21 / 95$ & 1 & 2 & 1 & 1 \\
\hline \hline $1 / 2$ & $10 / 93$ & $04 / 05 / 92-07 / 03 / 93$ & 1 & 2 & 2 & $1 / 1 / 1$ \\
\hline $1 / 2$ & $07 / 92$ & $03 / 31 / 91-04 / 04 / 92$ & 1 & 2 & 2 & $1 / 1 / 1$ \\
\hline \hline $1 / 2$ & $07 / 91$ & $04 / 01 / 90-03 / 30 / 91$ & 2 & 2 & 2 & $1 / 1 / 1$ \\
\hline \hline $1 / 2$ & $07 / 90$ & $07 / 01 / 89-03 / 31 / 90$ & 2 & 3 & 2 & $2 / 1 / 1$ \\
\hline \hline $1 / 2$ & $11 / 89$ & $05 / 01 / 88-06 / 30 / 89$ & 3 & 3 & 2 & $3 / 3 / 1$ \\
\hline
\end{tabular}

PLANT NAME: TURKEY POINT REGION: 2

\begin{tabular}{c|c|c|c|c|c|c|}
\multicolumn{1}{c}{ UNIT } & RPT & ASSESSMENT PERIOD & OPS & MAINT & ENG & PS $^{1}$ \\
\hline $3 / 4$ & $09 / 94$ & $01 / 31 / 93-08 / 27 / 94$ & 1 & 1 & 1 & 1 \\
\hline \hline $3 / 4$ & $05 / 93$ & $09 / 29 / 91-01 / 30 / 93$ & 1 & 2 & 2 & $1 / 1 / 1$ \\
\hline \hline $3 / 4$ & $12 / 91$ & $08 / 01 / 90-09 / 28 / 91$ & 1 & 2 & 2 & $2 / 1 / 1$ \\
\hline \hline $3 / 4$ & $10 / 90$ & $08 / 01 / 89-07 / 31 / 90$ & 2 & 2 & 2 & $2 / 2 / 2$ \\
\hline \hline $3 / 4$ & $12 / 89$ & $07 / 01 / 88-07 / 31 / 89$ & 2 & 2 & 2 & $2 / 2 / 3$ \\
\hline
\end{tabular}


(REACTORS IN OPERATION)

PLANT NAME: VOGTLE

REGION: 2

UNIT
\begin{tabular}{|c|c|c|c|c|c|c||}
\hline $1 / 2$ & RPT & ASSESSMENT PERIOD & OPS & MAINT & ENG & PS $^{1}$ \\
\hline \hline $1 / 2$ & $03 / 96$ & $07 / 03 / 94-01 / 27 / 96$ & 1 & 1 & 1 & 2 \\
\hline $1 / 2$ & $04 / 93$ & $09 / 29 / 91-01 / 02 / 93$ & 2 & 1 & 1 & $1 / 2 / 1$ \\
\hline \hline $1 / 2$ & $01 / 92$ & $10 / 01 / 90-09 / 28 / 91$ & 2 & 2 & 2 & $1 / 2 / 2$ \\
\hline \hline $1 / 2$ & $01 / 91$ & $10 / 01 / 89-09 / 30 / 90$ & 2 & 2 & 2 & $1 / 3 / 3$ \\
\hline \hline 1 & $01 / 90$ & $10 / 01 / 88-09 / 30 / 89$ & 2 & 1 & 2 & $2 / 2 / 2$ \\
\hline \hline 1 & $01 / 89$ & $10 / 01 / 87-09 / 30 / 88$ & 2 & 2 & 2 & $2 / 1 / 2$ \\
\hline
\end{tabular}

PLANT NAME: WATTS BAR ${ }^{6}$

REGION: 2

\begin{tabular}{|c|c|c|c|c|c|c|}
\hline UNIT & \multicolumn{1}{c}{ RPT } & ASSESSMENT PERIOD & OPS & MAINT & ENG & PS \\
\hline 1 & $\mathrm{XX} / \mathrm{XX}$ & $\mathrm{XX} / \mathrm{XX} / \mathrm{XX}-\mathrm{XX} / \mathrm{XX} / \mathrm{XX}$ & $\mathrm{X}$ & $\mathrm{X}$ & $\mathrm{X}$ & $\mathrm{X}$ \\
\hline
\end{tabular}

${ }^{6}$ Watts Bar recently received its operating 1 icense. The first Watts Bar SALP evaluation as an operating plant is scheduled to be performed in late 1996. 


\section{SALP HISTORY}

(REACTORS IN OPERATION)

PLANT NAME: BIG ROCK POINT

REGION: 3

\begin{tabular}{|c|c|c|c|c|c|c|}
\multicolumn{1}{c}{ UNIT } & RPT & ASSESSMENT PERIOD & OPS & MAINT & ENG & PS $^{1}$ \\
\hline \hline & $01 / 96$ & $05 / 01 / 94-11 / 25 / 95$ & 2 & 2 & 2 & 1 \\
\hline \hline & $06 / 94$ & $01 / 01 / 93-04 / 30 / 94$ & 2 & 2 & 2 & 2 \\
\hline \hline & $04 / 93$ & $10 / 01 / 91-12 / 31 / 92$ & 2 & 3 & 3 & $2 / 2 / 2$ \\
\hline \hline & $12 / 91$ & $05 / 01 / 90-09 / 30 / 91$ & 2 & 2 & 2 & $2 / 1 / 2$ \\
\hline \hline & $08 / 90$ & $01 / 01 / 89-04 / 30 / 90$ & 1 & 1 & 2 & $2 / 1 / 2$ \\
\hline \hline & $05 / 89$ & $09 / 01 / 87-12 / 31 / 88$ & 1 & 2 & 2 & $2 / 1 / 2$ \\
\hline
\end{tabular}

PLANT NAME: BRAIDWOOD

REGION: 3

UNIT
\begin{tabular}{|c|c|c|c|c|c|c||}
\hline \hline $1 / 2$ & RPT & ASSESSMENT PERIOD & OPS & MAINT & ENG & PS $^{1}$ \\
\hline \hline $1 / 2$ & $03 / 94$ & $02 / 20 / 94-09 / 30 / 95$ & 2 & 2 & 2 & 1 \\
\hline \hline $1 / 2$ & $12 / 92$ & $05 / 01 / 91-09 / 30 / 92$ & 2 & 1 & 2 & $2 / 2 / 1$ \\
\hline \hline $1 / 2$ & $07 / 91$ & $02 / 01 / 90-04 / 30 / 91$ & 2 & 1 & 2 & $2 / 1 / 1$ \\
\hline \hline $1 / 2$ & $05 / 90$ & $02 / 01 / 89-01 / 31 / 90$ & 2 & 1 & 2 & $2 / 1 / 2$ \\
\hline \hline $1 / 2$ & $06 / 89$ & $01 / 01 / 88-01 / 31 / 89$ & 2 & 2 & 2 & $2 / 1 / 2$ \\
\hline
\end{tabular}

NUREG-1214 
SALP HISTORY

\section{(REACTORS IN OPERATION)}

PLANT NAME: BYRON

REGION: 3

\begin{tabular}{|c|c|c|c|c|c|c|} 
UNIT & RPT & ASSESSMENT PERIOD & OPS & MAINT & ENG & PS $^{1}$ \\
\hline $1 / 2$ & $09 / 94$ & $03 / 01 / 93-08 / 20 / 94$ & 1 & 1 & 1 & 1 \\
\hline \hline $1 / 2$ & $05 / 93$ & $09 / 01 / 91-02 / 28 / 93$ & 1 & 1 & 2 & $1 / 1 / 1$ \\
\hline $1 / 2$ & $11 / 91$ & $04 / 01 / 90-08 / 31 / 91$ & 1 & 1 & 2 & $1 / 1 / 1$ \\
\hline $1 / 2$ & $07 / 90$ & $11 / 01 / 88-03 / 31 / 90$ & 1 & 1 & 2 & $1 / 1 / 1$ \\
\hline $1 / 2$ & $03 / 89$ & $11 / 01 / 87-10 / 31 / 88$ & 2 & 1 & 2 & $2 / 1 / 1$ \\
\hline
\end{tabular}

PLANT NAME: CLINTON

REGION: 3

\begin{tabular}{||c|c|c|c|c|c|c|} 
UNIT & RPT & ASSESSMENT PERIOD & OPS & MAINT & ENG & PS $^{1}$ \\
\hline & $08 / 95$ & $11 / 28 / 93-06 / 24 / 95$ & 2 & 1 & 2 & 1 \\
\hline & $01 / 94$ & $05 / 01 / 92-11 / 27 / 93$ & 1 & 1 & 2 & 2 \\
\hline \hline & $08 / 92$ & $02 / 01 / 91-04 / 30 / 92$ & 1 & 2 & 2 & $2 / 1 / 2$ \\
\hline & $06 / 91$ & $11 / 01 / 89-01 / 31 / 91$ & 2 & 2 & 2 & $2 / 1 / 2$ \\
\hline & $03 / 90$ & $09 / 01 / 88-10 / 31 / 89$ & 2 & 2 & 3 & $2 / 1 / 2$ \\
\hline & $01 / 89$ & $09 / 01 / 87-08 / 31 / 88$ & 2 & 2 & 2 & $2 / 2 / 1$ \\
\hline
\end{tabular}

NUREG-1214 


\section{SALP HISTORY}

\section{(REACTORS IN OPERATION)}

PLANT NAME: COOK

REGION: 3

\begin{tabular}{|c|c|c|c|c|c|c||}
\multicolumn{1}{c}{ UNIT } & RPT & ASSESSMENT PERIOD & OPS & MAINT & ENG & PS $^{1}$ \\
\hline $1 / 2$ & $07 / 96$ & $10 / 30 / 94-05 / 25 / 96$ & 2 & 2 & 2 & 1 \\
\hline \hline $1 / 2$ & $12 / 94$ & $05 / 01 / 93-10 / 29 / 94$ & 1 & 1 & 2 & 1 \\
\hline $1 / 2$ & $07 / 93$ & $01 / 01 / 92-04 / 30 / 93$ & 2 & 1 & 2 & $2 / 1 / 1$ \\
\hline $1 / 2$ & $05 / 92$ & $09 / 01 / 90-12 / 31 / 91$ & 2 & 2 & 2 & $2 / 1 / 1$ \\
\hline \hline $1 / 2$ & $12 / 90$ & $07 / 01 / 89-08 / 31 / 90$ & 1 & 3 & 3 & $2 / 1 / 1$ \\
\hline $1 / 2$ & $10 / 89$ & $03 / 01 / 88-06 / 30 / 89$ & 1 & 2 & 2 & $2 / 1 / 2$ \\
\hline
\end{tabular}

PLANT NAME: DAVIS-BESSE

REGION: 3

\begin{tabular}{||c|c|c|c|c|c|c||}
\multicolumn{1}{c}{ UNIT } & RPT & ASSESSMENT PERIOD & OPS & MAINT & ENG & PS $^{1}$ \\
\hline \hline & $03 / 95$ & $07 / 01 / 93-01 / 21 / 95$ & 1 & 1 & 1 & 1 \\
\hline \hline & $09 / 93$ & $12 / 01 / 91-06 / 30 / 93$ & 2 & 1 & 1 & $2 / 1 / 1$ \\
\hline \hline & $04 / 92$ & $07 / 01 / 90-11 / 30 / 91$ & 2 & 1 & 2 & $2 / 1 / 1$ \\
\hline \hline & $11 / 90$ & $03 / 01 / 89-06 / 30 / 90$ & 2 & 2 & 2 & $2 / 1 / 1$ \\
\hline & $07 / 89$ & $01 / 01 / 88-02 / 28 / 89$ & 2 & 2 & 2 & $2 / 1 / 1$ \\
\hline
\end{tabular}




\section{SALP HISTORY}

(REACTORS IN OPERATION)

PLANT NAME: DRESDEN

REGION: 3

\begin{tabular}{|c|c|c|c|c|c|c|}
\hline UNIT & RPT & ASSESSMENT PERIOD & OPS & MAINT & ENG & $\mathrm{PS}^{1}$ \\
\hline $2 / 3$ & $04 / 95$ & $08 / 22 / 93-03 / 18 / 95$ & 3 & 2 & 3 & 2 \\
\hline $2 / 3$ & $09 / 93$ & $08 / 01 / 92-08 / 21 / 93$ & 3 & 2 & 3 & 2 \\
\hline $2 / 3$ & $11 / 92$ & $08 / 01 / 91-07 / 31 / 92$ & 3 & 2 & 3 & $3 / 1 / 1$ \\
\hline $2 / 3$ & $11 / 91$ & $05 / 01 / 90-07 / 31 / 91$ & 2 & 2 & 2 & $2 / 1 / 1$ \\
\hline $2 / 3$ & $08 / 90$ & $02 / 01 / 89-04 / 30 / 90$ & 1 & 2 & 2 & $2 / 1 / 1$ \\
\hline $2 / 3$ & $05 / 89$ & $02 / 01 / 88-01 / 31 / 89$ & 1 & 2 & 2 & $2 / 1 / 2$ \\
\hline
\end{tabular}

PLANT NAME: DUANE ARNOLD

REGION: 3

\begin{tabular}{|c|c|c|c|c|c|c|}
\multicolumn{1}{c}{ UNIT } & RPT & ASSESSMENT PERIOD & OPS & MAINT & ENG & PS $^{1}$ \\
\hline \hline & $12 / 95$ & $03 / 20 / 94-10 / 28 / 95$ & 1 & 2 & 1 & 1 \\
\hline \hline & $04 / 94$ & $09 / 01 / 92-03 / 19 / 94$ & 1 & 2 & 2 & 1 \\
\hline \hline & $11 / 92$ & $04 / 01 / 91-08 / 31 / 92$ & 2 & 2 & 2 & $2 / 1 / 2$ \\
\hline \hline & $08 / 91$ & $01 / 01 / 90-03 / 31 / 91$ & 2 & 2 & 3 & $2 / 1 / 2$ \\
\hline \hline & $04 / 90$ & $11 / 01 / 88-12 / 31 / 89$ & 1 & 2 & 2 & $2 / 2 / 2$ \\
\hline \hline & $03 / 89$ & $06 / 01 / 87-10 / 31 / 88$ & 2 & 2 & 2 & $2 / 3 / 2$ \\
\hline
\end{tabular}

NUREG-1214 


\section{SALP HISTORY}

\section{(REACTORS IN OPERATION)}

PLANT NAME: FERMI

REGION: 3

\begin{tabular}{|c|c|c|c|c|c|c||}
\hline UNIT & RPT & ASSESSMENT PERIOD & OPS & MAINT & ENG & PS $^{1}$ \\
\hline 2 & $05 / 96$ & $04 / 03 / 94-03 / 30 / 96$ & 3 & 2 & 2 & 1 \\
\hline 2 & $05 / 94$ & $07 / 01 / 92-04 / 02 / 94$ & 3 & 2 & 2 & 1 \\
\hline 2 & $10 / 92$ & $03 / 01 / 91-06 / 30 / 92$ & 2 & 2 & 2 & $1 / 1 / 1$ \\
\hline 2 & $05 / 91$ & $01 / 01 / 90-02 / 28 / 91$ & 2 & 2 & 2 & $1 / 2 / 1$ \\
\hline 2 & $04 / 90$ & $01 / 01 / 89-12 / 31 / 89$ & 2 & 3 & 2 & $1 / 1 / 1$ \\
\hline 2 & $05 / 89$ & $04 / 01 / 88-12 / 31 / 88$ & 2 & 3 & 3 & $2 / 1 / 1$ \\
\hline
\end{tabular}

PLANT NAME: KEWAUNEE

REGION: 3

\begin{tabular}{||c|c|c|c|c|c|c||}
\multicolumn{1}{c}{ UNIT } & RPT & ASSESSMENT PERIOD & OPS & MAINT & ENG & PS $^{1}$ \\
\hline \hline & $03 / 95$ & $08 / 01 / 93-02 / 18 / 95$ & 1 & 1 & 1 & 1 \\
\hline \hline & $09 / 93$ & $03 / 01 / 92-07 / 31 / 93$ & 1 & 2 & 2 & 1 \\
\hline \hline & $06 / 92$ & $12 / 01 / 90-02 / 29 / 92$ & 1 & 2 & 2 & $1 / 1 / 2$ \\
\hline \hline & $04 / 91$ & $12 / 01 / 88-11 / 30 / 90$ & 1 & 1 & 2 & $1 / 1 / 2$ \\
\hline & $03 / 89$ & $07 / 01 / 87-11 / 30 / 88$ & 1 & 1 & 1 & $1 / 1 / 1$ \\
\hline
\end{tabular}




\section{SALP HISTORY}

\section{(REACTORS IN OPERATION)}

PLANT NAME: LASALLE

REGION: 3

\begin{tabular}{|c|c|c|c|c|c|c|}
\hline UNIT & RPT & ASSESSMENT PERIOD & OPS & MAINT & ENG & $\mathrm{PS}^{1}$ \\
\hline $1 / 2$ & $12 / 94$ & $06 / 01 / 93-11 / 26 / 94$ & 2 & 3 & 2 & 3 \\
\hline $1 / 2$ & $09 / 93$ & $01 / 01 / 92-05 / 31 / 93$ & 2 & 2 & 2 & $2 / 2 / 1$ \\
\hline $1 / 2$ & $03 / 92$ & $10 / 01 / 90-12 / 31 / 91$ & 1 & 2 & 2 & $2 / 1 / 1$ \\
\hline $1 / 2$ & $02 / 91$ & $07 / 01 / 89-09 / 30 / 90$ & 1 & 2 & 2 & $2 / 2 / 2$ \\
\hline $1 / 2$ & $10 / 89$ & $03 / 16 / 88-06 / 30 / 89$ & 1 & 2 & 2 & $2 / 1 / 2$ \\
\hline
\end{tabular}

PLANT NAME: MONTICELLO

REGION: 3

\begin{tabular}{|l|c|c|c|c|c|c||}
\multicolumn{1}{l}{ UNIT } & RPT & ASSESSMENT PERIOD & OPS & \multicolumn{1}{c}{ MAINT } & ENG & PS $^{1}$ \\
\hline \hline & $08 / 96$ & $12 / 25 / 94-07 / 20 / 96$ & 1 & 1 & 2 & 1 \\
\hline \hline & $01 / 95$ & $06 / 01 / 93-12 / 24 / 94$ & 2 & 1 & 1 & 1 \\
\hline \hline & $08 / 93$ & $12 / 01 / 91-05 / 31 / 93$ & 1 & 1 & 1 & $1 / 1 / 1$ \\
\hline \hline & $02 / 92$ & $07 / 01 / 90-11 / 30 / 91$ & 1 & 2 & 2 & $2 / 1 / 1$ \\
\hline \hline & $09 / 90$ & $03 / 01 / 89-06 / 30 / 90$ & 1 & 1 & 2 & $2 / 1 / 2$ \\
\hline \hline & $06 / 89$ & $12 / 01 / 87-02 / 28 / 89$ & 1 & 1 & 2 & $1 / 1 / 3$ \\
\hline
\end{tabular}




\section{SALP HISTORY}

\section{(REACTORS IN OPERATION)}

PLANT NAME: PALISADES

REGION: 3

\begin{tabular}{|c|c|c|c|c|c|c|}
\hline UNIT & RPT & ASSESSMENT PERIOD & OPS & MAINT & ENG & $\mathrm{PS}^{1}$ \\
\hline & $07 / 95$ & $10 / 31 / 93-05 / 27 / 95$ & 2 & $\underline{\underline{2}}$ & 2 & 2 \\
\hline & $12 / 93$ & $04 / 01 / 92-10 / 30 / 93$ & 3 & 2 & 3 & 2 \\
\hline & $07 / 92$ & $01 / 01 / 91-03 / 31 / 92$ & 1 & 1 & 2 & $2 / 2 / 1$ \\
\hline & $04 / 91$ & $09 / 01 / 89-12 / 31 / 90$ & 1 & 1 & 2 & $2 / 2 / 1$ \\
\hline & $01 / 90$ & $06 / 01 / 88-08 / 31 / 89$ & 2 & 2 & 2 & $2 / 1 / 1$ \\
\hline
\end{tabular}

PLANT NAME: PERRY

REGION: 3

\begin{tabular}{|c|c|c|c|c|c|c|}
\hline UNIT & RPT & ASSESSMENT PERIOD & OPS & MAINT & ENG & PS $^{1}$ \\
\hline 1 & $02 / 95$ & $02 / 01 / 93-01 / 07 / 95$ & 2 & 2 & 2 & 2 \\
\hline 1 & $04 / 93$ & $11 / 01 / 91-01 / 31 / 93$ & 2 & 2 & 2 & $2 / 1 / 1$ \\
\hline 1 & $02 / 92$ & $08 / 01 / 90-10 / 31 / 91$ & 2 & 2 & 2 & $2 / 1 / 1$ \\
\hline 1 & $11 / 90$ & $06 / 01 / 89-07 / 31 / 90$ & 2 & 2 & 2 & $2 / 1 / 1$ \\
\hline 1 & $10 / 89$ & $05 / 01 / 88-05 / 31 / 89$ & 2 & 2 & 2 & $2 / 1 / 1$ \\
\hline
\end{tabular}




\section{SALP HISTORY}

\section{(REACTORS IN OPERATION)}

PLANT NAME: POINT BEACH

REGION: 3

\begin{tabular}{|c|c|c|c|c|c|c||} 
UNIT & RPT & ASSESSMENT PERIOD & OPS & MAINT & ENG & PS $^{1}$ \\
\hline \hline $1 / 2$ & $06 / 96$ & $09 / 18 / 94-04 / 27 / 96$ & 2 & 2 & 2 & 1 \\
\hline $1 / 2$ & $10 / 94$ & $04 / 01 / 93-09 / 17 / 94$ & 2 & 2 & 2 & 1 \\
\hline $1 / 2$ & $07 / 93$ & $02 / 01 / 92-03 / 31 / 93$ & 2 & 2 & 2 & $1 / 1 / 2$ \\
\hline \hline $1 / 2$ & $05 / 92$ & $09 / 01 / 90-01 / 31 / 92$ & 1 & 2 & 2 & $2 / 1 / 2$ \\
\hline \hline $1 / 2$ & $01 / 91$ & $04 / 01 / 89-08 / 31 / 90$ & 1 & 1 & 2 & $2 / 2 / 2$ \\
\hline \hline $1 / 2$ & $07 / 89$ & $10 / 01 / 87-03 / 31 / 89$ & 2 & 1 & 2 & $1 / 2 / 2$ \\
\hline
\end{tabular}

PLANT NAME: PRAIRIE ISLAND

REGION: 3

\begin{tabular}{|c|c|c|c|c|c|c|}
\multicolumn{1}{c}{ UNIT } & RPT & ASSESSMENT PERIOD & OPS & MAINT & ENG & PS $^{1}$ \\
\hline $1 / 2$ & $03 / 96$ & $07 / 24 / 94-02 / 17 / 96$ & 1 & 1 & 2 & 1 \\
\hline \hline $1 / 2$ & $08 / 94$ & $02 / 01 / 93-07 / 23 / 94$ & 1 & 1 & 2 & 1 \\
\hline \hline $1 / 2$ & $04 / 93$ & $05 / 01 / 91-01 / 31 / 93$ & 1 & 1 & 2 & $1 / 1 / 2$ \\
\hline $1 / 2$ & $08 / 91$ & $05 / 01 / 89-04 / 30 / 91$ & 1 & 1 & 2 & $1 / 1 / 2$ \\
\hline $1 / 2$ & $08 / 89$ & $12 / 01 / 87-04 / 30 / 89$ & 1 & 1 & 2 & $1 / 1 / 2$ \\
\hline
\end{tabular}




\section{SALP HISTORY}

\section{(REACTORS IN OPERATION)}

PLANT NAME: QUAD CITIES

REGION: 3

\begin{tabular}{|c|c|c|c|c|c|c||}
\multicolumn{1}{c}{ UNIT } & RPT & ASSESSMENT PERIOD & OPS & MAINT & ENG & PS $^{1}$ \\
\hline $1 / 2$ & $08 / 95$ & $12 / 26 / 93-07 / 22 / 95$ & 2 & 3 & 2 & 2 \\
\hline \hline $1 / 2$ & $02 / 94$ & $06 / 01 / 92-12 / 25 / 93$ & 3 & 3 & 3 & 2 \\
\hline $1 / 2$ & $09 / 92$ & $03 / 01 / 91-05 / 31 / 92$ & 2 & 2 & 2 & $2 / 1 / 1$ \\
\hline \hline $1 / 2$ & $06 / 91$ & $12 / 01 / 89-02 / 28 / 91$ & 3 & 2 & 2 & $2 / 1 / 1$ \\
\hline \hline $1 / 2$ & $03 / 90$ & $10 / 01 / 88-11 / 30 / 89$ & 2 & 2 & 2 & $1 / 1 / 1$ \\
\hline \hline $1 / 2$ & $01 / 89$ & $04 / 01 / 87-09 / 30 / 88$ & 2 & 2 & 2 & $1 / 2 / 1$ \\
\hline
\end{tabular}

PLANT NAME: ZION

REGION: 3

\begin{tabular}{|c|c|c|c|c|c|c||}
\hline UNIT & RPT & ASSESSMENT PERIOD & OPS & MAINT & ENG & PS $^{1}$ \\
\hline $1 / 2$ & $02 / 96$ & $05 / 29 / 94-12 / 23 / 95$ & 3 & 2 & 2 & 2 \\
\hline \hline $1 / 2$ & $07 / 94$ & $12 / 01 / 92-05 / 28 / 94$ & 2 & 2 & 2 & 2 \\
\hline \hline $1 / 2$ & $02 / 93$ & $11 / 01 / 91-11 / 30 / 92$ & 2 & 2 & 2 & $2 / 1 / 1$ \\
\hline \hline $1 / 2$ & $01 / 92$ & $11 / 01 / 90-10 / 31 / 91$ & 2 & 3 & 2 & $2 / 2 / 2$ \\
\hline \hline $1 / 2$ & $03 / 91$ & $10 / 01 / 89-10 / 31 / 90$ & 3 & 3 & 3 & $2 / 2 / 2$ \\
\hline $1 / 2$ & $02 / 90$ & $06 / 01 / 88-09 / 30 / 89$ & 2 & 2 & 3 & $2 / 2 / 2$ \\
\hline
\end{tabular}

NUREG-1214 
PLANT NAME: ARKANSAS

REGION:

\begin{tabular}{|c|c|c|c|c|c|c|}
\hline UNIT & RPT & ASSESSMENT PERIOD & OPS & MAINT & ENG & $\mathrm{PS}^{1}$ \\
\hline $1 / 2$ & $08 / 96$ & $01 / 08 / 95-07 / 06 / 96$ & 1 & 2 & 1 & 1 \\
\hline $1 / 2$ & $02 / 95$ & $07 / 11 / 93-01 / 07 / 95$ & 1 & 1 & 2 & 1 \\
\hline $1 / 2$ & $11 / 93$ & $03 / 01 / 92-07 / 10 / 93$ & 1 & 2 & 2 & $2 / 1 / 1$ \\
\hline $1 / 2$ & $06 / 92$ & $12 / 01 / 90-02 / 29 / 92$ & 1 & 2 & 2 & $2 / 1 / 1$ \\
\hline $1 / 2$ & $04 / 91$ & $10 / 01 / 89-11 / 30 / 90$ & 2 & 2 & 2 & $2 / 1 / 1$ \\
\hline $1 / 2$ & $02 / 90$ & $07 / 01 / 88-09 / 30 / 89$ & $\underline{2}$ & 3 & 2 & $2 / 1 / 2$ \\
\hline $1 / 2$ & $03 / 89$ & $01 / 01 / 87-06 / 30 / 88$ & 1 & 2 & 2 & $1 / 1 / 2$ \\
\hline
\end{tabular}

PLANT NAME: CALLAWAY

REGION:

\begin{tabular}{|c|c|c|c|c|c|c|}
\hline UNIT & RPT & ASSESSMENT PERIOD & OPS & MAINT & ENG & $\mathrm{PS}^{1}$ \\
\hline & $06 / 95$ & $09 / 19 / 93-04 / 29 / 95$ & 1 & 1 & 1 & 1 \\
\hline & $10 / 93$ & $02 / 01 / 92-09 / 18 / 93$ & 1 & 1 & 1 & 2 \\
\hline & $05 / 92$ & $02 / 01 / 90-01 / 31 / 92$ & 1 & 1 & 1 & $2 / 1 / 1$ \\
\hline & $05 / 90$ & $09 / 01 / 88-01 / 31 / 90$ & 1 & 1 & 1 & $1 / 1 / 1$ \\
\hline & $12 / 88$ & $06 / 01 / 87-08 / 31 / 88$ & 2 & 1 & 2 & $2 / 1 / 1$ \\
\hline
\end{tabular}

NUREG-1214 


\section{SALP HISTORY}

\section{(REACTORS IN OPERATION)}

PLANT NAME: COMANCHE PEAK

REGION: 4

\begin{tabular}{|c|c|c|c|c|c|c|}
\hline UNIT & RPT & ASSESSMENT PERIOD & OPS & MAINT & ENG & PS $^{9}$ \\
\hline $1 / 2$ & $12 / 95$ & $05 / 29 / 94-11 / 25 / 95$ & 1 & 1 & 2 & 1 \\
\hline $1 / 2$ & $06 / 94$ & $05 / 30 / 93-05 / 28 / 94$ & 2 & 2 & 1 & 1 \\
\hline $1 / 2$ & $08 / 93$ & $02 / 03 / 92-05 / 29 / 93$ & 2 & 2 & 1 & $1 / 1 / 2$ \\
\hline 1 & $05 / 92$ & $02 / 03 / 91-02 / 01 / 92$ & 2 & 2 & 1 & $1 / 1 / 1$ \\
\hline 1 & $05 / 91$ & $09 / 01 / 89-01 / 31 / 91$ & 2 & 2 & 2 & $2 / 1 / 1$ \\
\hline
\end{tabular}

PLANT NAME: COOPER

REGION: 4

\begin{tabular}{|c|c|c|c|c|c|c|}
\hline UNIT & RPT & ASSESSMENT PERIOD & OPS & MAINT & ENG & $\mathrm{PS}^{1}$ \\
\hline & $08 / 95$ & $04 / 25 / 93-07 / 08 / 95$ & 2 & 3 & 3 & 2 \\
\hline & $08 / 93$ & $01 / 19 / 92-04 / 24 / 93$ & 2 & 3 & 2 & $2 / 2 / 1$ \\
\hline & $04 / 92$ & $07 / 16 / 90-01 / 18 / 92$ & $\underline{2}$ & 1 & 2 & $2 / 2 / 1$ \\
\hline & $12 / 90$ & $04 / 16 / 89-07 / 15 / 90$ & 1 & 2 & 2 & $1 / 2 / 2$ \\
\hline & $08 / 89$ & $02 / 01 / 88-04 / 15 / 89$ & 1 & 2 & 2 & $1 / 2 / 2$ \\
\hline
\end{tabular}

NUREG-1214 


\section{SALP HISTORY}

\section{(REACTORS IN OPERATION)}

PLANT NAME: DIABLO CANYON REGION: 4

\begin{tabular}{|c|c|c|c|c|c|c||}
\multicolumn{1}{c}{ UNIT } & RPT & ASSESSMENT PERIOD & OPS & MAINT & ENG & PS $^{1}$ \\
\hline \hline $1 / 2$ & $09 / 96$ & $09 / 01 / 94-08 / 17 / 96$ & 2 & 2 & 2 & 1 \\
\hline \hline $1 / 2$ & $09 / 94$ & $01 / 01 / 93-08 / 31 / 94$ & 1 & 1 & 1 & 1 \\
\hline \hline $1 / 2$ & $03 / 93$ & $07 / 01 / 91-12 / 31 / 92$ & 1 & 1 & 1 & $1 / 1 / 2$ \\
\hline \hline $1 / 2$ & $09 / 91$ & $01 / 01 / 90-06 / 30 / 91$ & 1 & 2 & 1 & $1 / 2 / 2$ \\
\hline \hline $1 / 2$ & $05 / 90$ & $08 / 01 / 88-12 / 31 / 89$ & 1 & 2 & 2 & $1 / 1 / 2$ \\
\hline \hline $1 / 2$ & $01 / 89$ & $08 / 01 / 87-07 / 31 / 88$ & 2 & 2 & 2 & $1 / 1 / 2$ \\
\hline
\end{tabular}

PLANT NAME: FORT CALHOUN REGION: 4

\begin{tabular}{|c|c|c|c|c|c|c|}
\hline UNIT & RPT & ASSESSMENT PERIOD & OPS & MAINT & ENG & $\mathrm{PS}^{1}$ \\
\hline & $03 / 96$ & $07 / 31 / 94-01 / 27 / 96$ & 2 & 2 & 1 & 1 \\
\hline & $08 / 94$ & $01 / 31 / 93-07 / 30 / 94$ & 2 & 2 & 1 & 1 \\
\hline & $05 / 93$ & $08 / 01 / 91-01 / 30 / 93$ & 2 & 2 & 1 & $2 / 1 / 1$ \\
\hline & $11 / 91$ & $05 / 01 / 90-07 / 31 / 91$ & 2 & 2 & 1 & $1 / 2 / 1$ \\
\hline & $09 / 90$ & $05 / 01 / 89-04 / 30 / 90$ & 2 & 2 & 2 & $1 / 2 / 2$ \\
\hline & $08 / 89$ & $05 / 01 / 88-04 / 30 / 89$ & 2 & 2 & 2 & $2 / 2 / 2$ \\
\hline
\end{tabular}




\section{SALP HISTORY}

\section{(REACTORS IN OPERATION)}

PLANT NAME: GRAND GULF

REGION:

\begin{tabular}{|c|c|c|c|c|c|c||}
\hline UNIT & RPT & ASSESSMENT PERIOD & OPS & MAINT & ENG & PS $^{1}$ \\
\hline 1 & $03 / 96$ & $02 / 27 / 94-02 / 24 / 96$ & 1 & 2 & 1 & 1 \\
\hline \hline 1 & $04 / 94$ & $08 / 23 / 92-02 / 26 / 94$ & 1 & 1 & 1 & 1 \\
\hline \hline 1 & $12 / 92$ & $02 / 24 / 91-08 / 22 / 92$ & 1 & 1 & 2 & $1 / 1 / 1$ \\
\hline \hline 1 & $05 / 91$ & $10 / 01 / 89-02 / 23 / 91$ & 1 & 1 & 1 & $1 / 1 / 1$ \\
\hline \hline 1 & $01 / 90$ & $05 / 01 / 88-09 / 30 / 89$ & 1 & 1 & 2 & $1 / 1 / 1$ \\
\hline
\end{tabular}

PLANT NAME: PALO VERDE

REGION: 4

\begin{tabular}{||c|c|c|c|c|c|c||}
\hline UNIT & RPT & ASSESSMENT PERIOD & OPS & MAINT & ENG & PS $^{1}$ \\
\hline $1 / 2 / 3$ & $07 / 96$ & $12 / 01 / 94-06 / 01 / 96$ & 1 & 1 & 1 & 2 \\
\hline $1 / 2 / 3$ & $01 / 95$ & $06 / 01 / 93-11 / 30 / 94$ & 2 & 2 & 2 & 2 \\
\hline $1 / 2 / 3$ & $10 / 93$ & $03 / 01 / 92-05 / 31 / 93$ & 2 & 2 & 2 & $2 / 1 / 2$ \\
\hline $1 / 2 / 3$ & $07 / 92$ & $12 / 01 / 90-02 / 29 / 92$ & 2 & 2 & 2 & $1 / 1 / 2$ \\
\hline \hline $1 / 2 / 3$ & $03 / 91$ & $11 / 01 / 89-11 / 30 / 90$ & 2 & 2 & 2 & $2 / 1 / 2$ \\
\hline $1 / 2 / 3$ & $01 / 90$ & $11 / 01 / 88-10 / 31 / 89$ & 2 & 3 & 3 & $2 / 2 / 2$ \\
\hline $1 / 2 / 3$ & $03 / 89$ & $11 / 01 / 87-10 / 31 / 88$ & 3 & 2 & 2 & $3 / 2 / 2$ \\
\hline \hline
\end{tabular}

NUREG-1214 


\section{SALP HISTORY}

(REACTORS IN OPERATION)

PLANT NAME: RIVER BEND

REGION: 4

\begin{tabular}{l|c|c|c|c|c|c|c|} 
UNIT & RPT & ASSESSMENT PERIOD & OPS & MAINT & ENG & PS $^{1}$ \\
\hline \hline 1 & $08 / 95$ & $01 / 30 / 94-07 / 29 / 95$ & 2 & 2 & 2 & 1 \\
\hline 1 & $03 / 94$ & $09 / 27 / 92-01 / 29 / 94$ & 2 & 3 & 3 & 2 \\
\hline 1 & $01 / 93$ & $04 / 01 / 91-09 / 26 / 92$ & 2 & 2 & 2 & $2 / 1 / 2$ \\
\hline \hline 1 & $08 / 91$ & $01 / 01 / 90-03 / 31 / 91$ & 1 & 2 & 2 & $2 / 1 / 2$ \\
\hline 1 & $05 / 90$ & $10 / 01 / 88-12 / 31 / 89$ & 1 & 2 & 2 & $1 / 1 / 2$ \\
\hline 1 & $03 / 89$ & $04 / 01 / 87-09 / 30 / 88$ & 2 & 2 & 1 & $2 / 1 / 3$ \\
\hline
\end{tabular}

PLANT NAME: SAN ONOFRE

REGION: 4

\begin{tabular}{|c|c|c|c|c|c|c|}
\hline UNIT & RPT & ASSESSMENT PERIOD & OPS & MAINT & ENG & $\mathrm{PS}^{1}$ \\
\hline $2 / 3$ & $02 / 96$ & $07 / 01 / 94-12 / 30 / 95$ & 2 & 2 & 1 & 1 \\
\hline $2 / 3$ & $08 / 94$ & $12 / 01 / 92-06 / 30 / 94$ & 1 & 2 & 2 & 1 \\
\hline $1 / 2 / 3$ & $03 / 93$ & $08 / 01 / 91-11 / 30 / 92$ & 1 & 2 & 2 & $1 / 1 / 1$ \\
\hline $1 / 2 / 3$ & $11 / 91$ & $02 / 01 / 90-07 / 31 / 91$ & 2 & 2 & 2 & $1 / 1 / 1$ \\
\hline $1 / 2 / 3$ & $05 / 90$ & $10 / 01 / 88-01 / 31 / 90$ & 2 & 1 & 2 & $1 / 1 / 1$ \\
\hline $1 / 2 / 3$ & $01 / 89$ & $10 / 01 / 87-09 / 30 / 88$ & 1 & 2 & 3 & $1 / 1 / 1$ \\
\hline
\end{tabular}




\section{SALP HISTORY}

(REACTORS IN OPERATION)

PLANT NAME: SOUTH TEXAS

REGION: 4

\begin{tabular}{||c|c|c|c|c|c|c||}
\multicolumn{1}{c}{ UNIT } & RPT & ASSESSMENT PERIOD & OPS & MAINT & ENG & PS $^{1}$ \\
\hline $1 / 2$ & $05 / 96$ & $09 / 25 / 94-03 / 23 / 96$ & 2 & 1 & 2 & 1 \\
\hline \hline $1 / 2$ & $10 / 94$ & $08 / 02 / 92-09 / 24 / 94$ & 2 & 2 & 2 & 2 \\
\hline $1 / 2$ & $11 / 92$ & $06 / 02 / 91-08 / 01 / 92$ & 2 & 2 & 2 & $1 / 2 / 2$ \\
\hline $1 / 2$ & $09 / 91$ & $02 / 01 / 90-05 / 31 / 91$ & 2 & 2 & 2 & $1 / 2 / 1$ \\
\hline $1 / 2$ & $06 / 90$ & $01 / 01 / 89-01 / 31 / 90$ & 1 & 1 & 2 & $2 / 2 / 1$ \\
\hline $1 / 2$ & $05 / 89$ & $01 / 01 / 88-12 / 31 / 88$ & 2 & 2 & 2 & $2 / 2 / 2$ \\
\hline
\end{tabular}

PLANT NAME: WASHINGTON NUCLEAR

REGION: 4

\begin{tabular}{|c|c|c|c|c|c|c|}
\hline UNIT & RPT & ASSESSMENT PERIOD & OPS & MAINT & ENG & $\mathrm{PS}^{1}$ \\
\hline 2 & $09 / 95$ & $04 / 01 / 94-09 / 02 / 95$ & 3 & 2 & 3 & 2 \\
\hline 2 & $05 / 94$ & $03 / 01 / 93-03 / 31 / 94$ & 2 & 2 & 3 & 2 \\
\hline 2 & $06 / 93$ & $01 / 01 / 92-02 / 28 / 93$ & 2 & 2 & 3 & $2 / 2 / 1$ \\
\hline 2 & $03 / 92$ & $09 / 01 / 90-12 / 31 / 91$ & 3 & 2 & 2 & $2 / 2 / 1$ \\
\hline 2 & $12 / 90$ & $06 / 01 / 89-08 / 31 / 90$ & 1 & 2 & 2 & $2 / 2 / 2$ \\
\hline 2 & $10 / 89$ & $06 / 01 / 88-05 / 31 / 89$ & 2 & 2 & 2 & $2 / 1 / 2$ \\
\hline 2 & $10 / 88$ & $06 / 01 / 87-05 / 31 / 88$ & 3 & 2 & 3 & $2 / 2 / 1$ \\
\hline
\end{tabular}

NUREG-1214 
SALP HISTORY

(REACTORS IN OPERATION)

PLANT NAME: WATERFORD

REGION:

\begin{tabular}{|c|c|c|c|c|c|c|}
\hline UNIT & RPT & ASSESSMENT PERIOD & OPS & MAINT & ENG & $\mathrm{PS}^{1}$ \\
\hline 3 & $05 / 95$ & $10 / 31 / 93-04 / 29 / 95$ & 2 & 1 & 1 & 1 \\
\hline 3 & $12 / 93$ & $08 / 02 / 92-10 / 30 / 93$ & 2 & 1 & 2 & 1 \\
\hline 3 & $11 / 92$ & $05 / 01 / 91-08 / 01 / 92$ & 1 & 1 & 2 & $1 / 1 / 1$ \\
\hline 3 & $08 / 91$ & $11 / 01 / 89-04 / 30 / 91$ & 1 & 2 & 2 & $1 / 1 / 1$ \\
\hline 3 & $03 / 90$ & $08 / 01 / 88-10 / 31 / 89$ & 1 & 2 & 2 & $1 / 1 / 1$ \\
\hline 3 & $02 / 89$ & $02 / 01 / 87-07 / 31 / 88$ & 2 & 2 & 2 & $2 / 1 / 1$ \\
\hline
\end{tabular}

PLANT NAME: WOLF CREEK

REGION: 4

\begin{tabular}{|c|c|c|c|c|c|c||}
\multicolumn{1}{c}{ UNIT } & \multicolumn{1}{c}{ RPT } & ASSESSMENT PERIOD & OPS & \multicolumn{1}{c|}{ MAINT } & ENG & PS $^{1}$ \\
\hline \hline 1 & $11 / 95$ & $04 / 10 / 94-10 / 07 / 95$ & 1 & 2 & 2 & 1 \\
\hline \hline 1 & $05 / 94$ & $10 / 11 / 92-04 / 09 / 94$ & 2 & 2 & 2 & 2 \\
\hline \hline 1 & $01 / 93$ & $10 / 06 / 91-10 / 10 / 92$ & 2 & 2 & 2 & $1 / 2 / 1$ \\
\hline 1 & $01 / 92$ & $07 / 01 / 90-10 / 05 / 91$ & 2 & 2 & 2 & $2 / 1 / 1$ \\
\hline \hline 1 & $10 / 90$ & $04 / 01 / 89-06 / 30 / 90$ & 2 & 2 & 2 & $2 / 1 / 1$ \\
\hline 1 & $07 / 89$ & $04 / 01 / 88-03 / 31 / 89$ & 2 & 2 & 2 & $2 / 1 / 1$ \\
\hline
\end{tabular}

NUREG-1214 
NRC FORM 335

(2-89)

NRCM 1102

3201,3202

\section{TITLE AND SUBTITLE}

Historical Data Summary of the Systematic Assessment of Licensee Performance
U.S. NUCLEAR REGULATORY COMMISSION

BIBLIOGRAPHIC DATA SHEET

(Seo instructions on the reverse)
1. REPORT NUMBER

(Assigned by NRC, Add Vol., Supp., Rev., and Addendum Numbers, if any.)

\section{NUREG-1214}

Revision 14

3. DATE REPORT PUBLISHED

\begin{tabular}{l|l} 
MONTH & YEAR
\end{tabular}

October 1996

4. FIN OR GRANT NUMBER

6. TYPE OF REPORT

5. AUTHOR(S)

D. Gamberoni

7. PERIOD COVERED (Inclusive Dates)

$01 / 01 / 94-09 / 30 / 96$

8. PERFORMING ORGANIZATION - NAME AND ADDRESS (If NRC, provide Division, Office or Region, U.S. Nuclear Regulatory Commission, and mailing address; if contractor, provide name and mailing address.)

Division of Inspection and Support Programs

Office of Nuclear Reactor Regulation

U.S. Nuclear Reactory Commission

Washington, DC 20555 - 0001

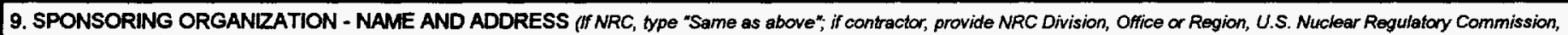
and mailing address.)

Same as above

10. SUPPLEMENTARY NOTES

11. ABSTRACT (200 words or loss)

The Historical Data Summary of the Systematic Assessment of Licensee Performance (SALP) is produced periodically by the U.S.

Nuclear Regulatory Commission. This summary provides the results of the assessment of each facility by NRC region and is

further divided into the following sections: Section 1 presents the most recent SALP report ratings for facilities in operation.

Section 2 presents a chronological listing of all SALP report ratings for each operating facility since August 1, 1988.

12. KEY WORDSIDESCRIPTORS (List words or phrases thet will assist researchers in locating the report.)

Systematic Assessment of Licensee Performance (SALP)

U.S. Nuclear Power Plants

Licensees

Operating Facilities

Performance History

\begin{tabular}{|c|}
\hline $\begin{array}{l}\text { 13. AVAILABILITY STATEMENT } \\
\text { Unlimited }\end{array}$ \\
\hline 14. SECURITY CLASSIFICATION \\
\hline (This Page) \\
\hline Unclassified \\
\hline $\begin{array}{l}\text { (This Report) } \\
\text { Unclassified }\end{array}$ \\
\hline 15. NUMBER OF PAGES \\
\hline 16. PRICE \\
\hline
\end{tabular}

